\title{
Multi-Omics Analysis of Fatty Alcohol Production in Engineered Yeasts Saccharomyces cerevisiae and Yarrowia lipolytica
}

\section{OPEN ACCESS \\ Edited by: \\ Isabel Sá-Correia, \\ University of Lisbon, \\ Portugal \\ Reviewed by: \\ Jennifer Gallagher, \\ West Virginia University, \\ United States \\ Pau Ferrer, \\ Autonomous University of Barcelona,}

Spain

*Correspondence:

Irina Borodina

irbo@biosustain.dtu.dk

tPresent address:

Birgitta E. Ebert,

Vickers Group, Australian Institute for Bioengineering and Nanotechnology, Brisbane City,

QLD, Australia

Specialty section:

This article was submitted to

Evolutionary and Genomic

Microbiology,

a section of the journal

Frontiers in Genetics

Received: 15 April 2019

Accepted: 17 July 2019

Published: 30 August 2019

Citation:

Dahlin J, Holkenbrink C, Marella ER, Wang G, Liebal U, Lieven C, Weber D, McCloskey D, Wang $H-L$, Ebert BE, Herrgård MJ,

Blank LM and Borodina I

(2019) Multi-Omics Analysis

of Fatty Alcohol Production in

Engineered Yeasts Saccharomyces cerevisiae and Yarrowia lipolytica.

Front. Genet. 10:747.

doi: 10.3389/fgene.2019.00747

\begin{abstract}
Jonathan Dahlin ${ }^{1}$, Carina Holkenbrink', Eko Roy Marella', Guokun Wang ${ }^{1}$, Ulf Liebal' ${ }^{2}$, Christian Lieven ${ }^{1}$, Dieter Weber ${ }^{2}$, Douglas McCloskey ${ }^{1}$, Hong-Lei Wang ${ }^{3}$, Birgitta E. Ebert ${ }^{2 t}$, Markus J. Herrgård ${ }^{1}$, Lars Mathias Blank ${ }^{2}$ and Irina Borodina ${ }^{1 *}$

${ }^{1}$ The Novo Nordisk Foundation Center for Biosustainability, Technical University of Denmark, Kongens Lyngby, Denmark, ${ }^{2}$ AMB - Institute of Applied Microbiology, ABBt - Aachen Biology and Biotechnology, RWTH Aachen University, Aachen, Germany, ${ }^{3}$ Department of Biology, Lund University, Lund, Sweden
\end{abstract}

Fatty alcohols are widely used in various applications within a diverse set of industries, such as the soap and detergent industry, the personal care, and cosmetics industry, as well as the food industry. The total world production of fatty alcohols is over 2 million tons with approximately equal parts derived from fossil oil and from plant oils or animal fats. Due to the environmental impact of these production methods, there is an interest in alternative methods for fatty alcohol production via microbial fermentation using cheap renewable feedstocks. In this study, we aimed to obtain a better understanding of how fatty alcohol biosynthesis impacts the host organism, baker's yeast Saccharomyces cerevisiae or oleaginous yeast Yarrowia lipolytica. Producing and non-producing strains were compared in growth and nitrogen-depletion cultivation phases. The multi-omics analysis included physiological characterization, transcriptome analysis by RNAseq, ${ }^{13} \mathrm{C}$ metabolic flux analysis, and intracellular metabolomics. Both species accumulated fatty alcohols under nitrogen-depletion conditions but not during growth. The fatty alcohol-producing $Y$. lipolytica strain had a higher fatty alcohol production rate than an analogous S. cerevisiae strain. Nitrogen-depletion phase was associated with lower glucose uptake rates and a decrease in the intracellular concentration of acetyl-CoA in both yeast species, as well as increased organic acid secretion rates in $Y$. lipolytica. Expression of the fatty alcohol-producing enzyme fatty acyl-CoA reductase alleviated the growth defect caused by deletion of hexadecenal dehydrogenase encoding genes (HFD1 and HFD4) in Y. lipolytica. RNAseq analysis showed that fatty alcohol production triggered a cell wall stress response in S. cerevisiae. RNAseq analysis also showed that both nitrogen-depletion and fatty alcohol production have substantial effects on the expression of transporter encoding genes in Y. lipolytica. In conclusion, through this multi-omics study, we uncovered some effects of fatty alcohol production on the host metabolism. This knowledge can be used as guidance for further strain improvement towards the production of fatty alcohols.

Keywords: fatty alcohol, metabolome, ${ }^{13} \mathrm{C}$-fluxome, transcriptome, Yarrowia lipolytica, Saccharomyces cerevisiae 


\section{INTRODUCTION}

Fatty alcohols are used as detergents and surfactants in personal care products such as soaps, shampoos, or creams. The global market for fatty alcohols is estimated at 5 billion USD (Grand View Research, Inc. 2016). The major fraction of the fatty alcohols used today are derived from either crude oil or palm kernel oil, both feedstocks being non-sustainable on a long term (Shah et al., 2016). Alternatively, fatty alcohols can be produced from abundant renewable feedstocks via microbial fermentation.

Some marine bacteria can produce fatty alcohols naturally; however, the titers are low, and these organisms are not suitable for large-scale fermentation. Hence, several industrially applicable hosts have been engineered to produce fatty alcohols. Escherichia coli was engineered to produce $6.3 \mathrm{~g} / \mathrm{L}$ total fatty alcohols (Liu et al., 2016) (bioreactor, complex media). Baker's yeast Saccharomyces cerevisiae was engineered to achieve a titer of $6.0 \mathrm{~g} / \mathrm{L}$ (d'Espaux et al., 2017) (bioreactor, complex media). Several oleaginous yeast species have also applied for fatty alcohol production. Yarrowia lipolytica has been engineered to produce $2.2 \mathrm{~g} / \mathrm{L}$ (Xu et al., 2016) (bioreactor, minimal media), and Lipomyces starkeyi was engineered to produce $1.7 \mathrm{~g} / \mathrm{L}$ (McNeil and Stuart 2018) (shake flask, minimal media). To this date, the highest reported titer of $8 \mathrm{~g} / \mathrm{L}$ total alcohols was obtained with oleaginous yeast Rhodosporidium toruloides (Fillet et al., 2015) (bioreactor, complex media).

Fatty alcohol biosynthesis is carried out in two enzymatic steps from fatty acyl-CoAs, key intermediates in membrane, and storage lipid biosynthesis. The two enzymatic steps can be carried out by two enzymes, an aldehyde-forming longchain acyl-CoA reductase (ACR, EC 1.2.1.50), and an aldehyde reductase (AHR, EC 1.1.1.2), where ACR converts the fatty acylCoA into a fatty aldehyde, which in turn is converted by AHR into fatty alcohol. The two enzymatic steps can also be carried out by a single enzyme, an alcohol-forming fatty acyl-CoA reductase (FAR, EC 1.2.1.84), where FAR converts fatty acyl-CoA into fatty alcohol, with a fatty aldehyde as a transient intermediate. The conversion of fatty acyl-CoA into corresponding fatty alcohol requires two NADPH molecules (Figure 1).
The process performance parameters reported for fatty alcohols in the literature do not yet meet the requirements for industrial production of bulk chemicals. Further strain improvement and process optimization are required. A better understanding of the effect of fatty alcohol production on a cell factory can guide further strain improvement. In this study, we performed a multi-omics analysis comparing gene expression, fluxes, and intracellular metabolites' concentrations in fatty alcoholproducing S. cerevisiae and Y. lipolytica strains.

\section{MATERIALS AND METHODS}

\section{Strains, Reagents, and Chemicals}

Escherichia coli DH5 $\alpha$ was used for manipulation of DNA during cloning. S. cerevisiae CEN.PK113-7D (MATa URA3 HIS3 LEU2 TRP1 MAL2-8c SUC2) was a gift from Dr. Peter Kötter (GoetheUniversität, Germany). Y. lipolytica GB20 (MATb, ku70 nugm-Htg2, ndh2i, lys11-, leu2-, ura3-) was a gift from Prof. Volker Zickermann (Goethe-Universität, Germany). Cloning reagents were sourced according to the EasyClone-Markerfree (Jessop-Fabre et al., 2016) and EasyCloneYALI (Holkenbrink et al., 2018). All chemicals were acquired from Sigma-Aldrich unless otherwise specified. Nourseothricin was acquired from Jena Bioscience GmbH (Germany).

\section{Strain Construction}

The S. cerevisiae strains were constructed using the EasyCloneMarkerFree toolbox (Jessop-Fabre et al., 2016). The fatty alcohol degradation-deficient strain (ST6849) was constructed from CEN.PK113-7D by introducing a Cas9 plasmid pCfB2312, followed by a knock out of the PEX10 and HFD1 genes using the single guide RNA (sgRNA) plasmid and repair templates (synthesized DNA fragment) as described in Supplementary Table S1. The fatty alcohol-producing strain (ST6989) was constructed by expressing a total of four copies of the fatty acylCoA reductase from Marinobacter algicola (malFAR), codonoptimized for Y. lipolytica (sequence detailed in Supplementary

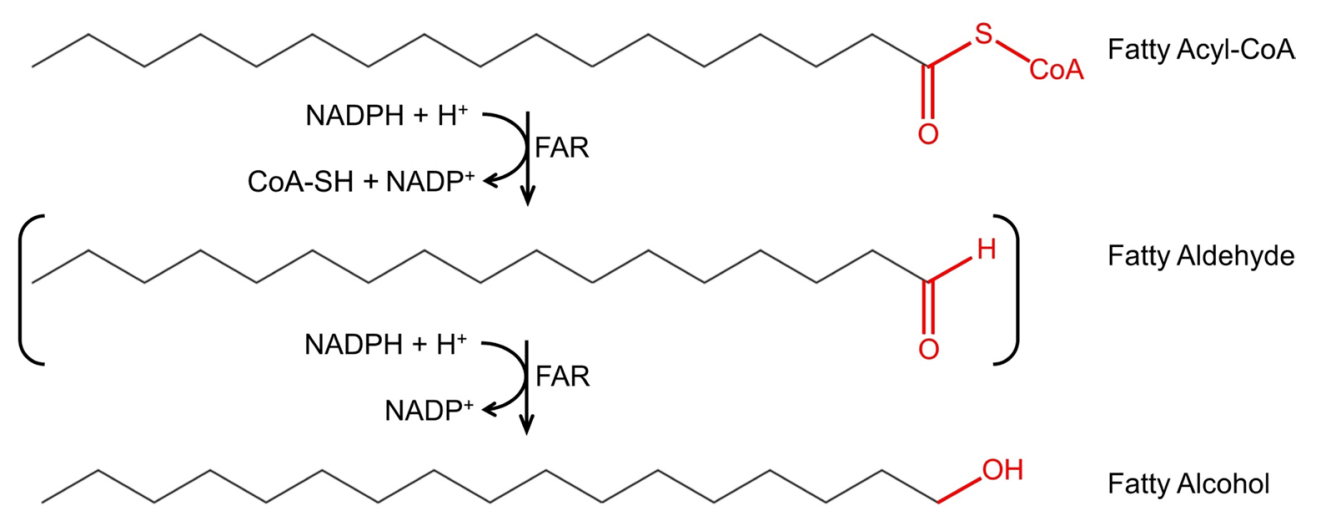

FIGURE 1 | Fatty alcohol biosynthesis via fatty acyl-CoA reductase (FAR). FAR catalyzes the conversion of fatty acyl-CoAs to fatty alcohols with the fatty aldehyde as transient intermediate (indicated as brackets). The substrate depicted in the figure is hexadecanoyl-CoA; however, carbon chain length and saturation may vary. 
File S1), in the degradation-deficient strain. Integration vectors containing two copies of malFAR were constructed, consisting of one copy of malFAR under the TDH3 (GPD) promoter and one copy under the TEF1 promoter, which were inserted into vectors $\mathrm{pCfB} 3034$ and $\mathrm{pCfB} 3037$ to generate vectors $\mathrm{pCfB} 7082$ and pCfB7083, respectively (Supplementary Table S1). The NotI-digested plasmids were inserted into integration sites X-3 and XI-5, using the double sgRNA plasmid pCfB5283.

The Y. lipolytica degradation-deficient strain (ST6770) was constructed from strain ST6276 (Borodina et al., 2018). ST6276 is derived from Y. lipolytica strain GB20 by deleting genes FAO1, HFD1, HFD4, and PEX10. The leucine and uracil auxotrophies were closed by integrating an expression cassette containing URA3 and LEU2 using vector pCfB7093 and selecting for growth on SC-Ura-Leu agar plates. The lysine auxotrophy was closed by restoring the native sequence of the homocitrate synthase gene (YALI1_F38776g) using DNA fragment BB2251 and selecting for growth on SC-Lys agar plates. The fatty alcoholproducing strain (ST6987) was constructed by expressing a total of four copies of the malFAR, codon-optimized for Y. lipolytica, in the degradation-deficient strain ST6770. A two-gene construct, consisting of one copy of malFAR under the GPD promoter and one copy under the TEF-intron promoter, were inserted into vector pCfB4796 and $\mathrm{pCfB} 4784$ to generate plasmid pCfB7091 and pCfB7092, respectively (Supplementary Table S1). The NotI-digested plasmids were inserted at both integration sites D-1 and F-2.

The promoter and coding sequences of all vectors were verified using Sanger sequencing provided by Eurofins Genomics, and integration was verified by colony PCR.

\section{Cultivation}

Unless otherwise specified, all cultures were grown in baffled shake flasks equipped with caps containing air-permeable membranes, incubated at $30^{\circ} \mathrm{C}$ at 250 rotations per minute (RPM) in a MaxQ 8000 Orbital Shaker (Thermo Fisher). Precultures were grown on minimal media until the late exponential phase, and subsequently washed and resuspended in either fresh minimal media or nitrogen-depleted media for growth phase or nitrogen-depleted stationary phase studies respectively. For the pre-cultures, the media contained $20 \mathrm{~g} / \mathrm{L}$ glucose, $5 \mathrm{~g} / \mathrm{L}$ ammonium sulfate, $12 \mathrm{~g} / \mathrm{L}$ potassium phosphate $(\mathrm{pH} 6.0), 3.4 \mathrm{~g} / \mathrm{L}$ yeast nitrogen base (YNB, w/o amino acids, w/o ammonium sulfate), and 1\% YPD. Pre-cultures were centrifuged at 5,000 $\times \mathrm{g}$ for $5 \mathrm{~min}$ at room temperature and washed with an equal volume of sterile water; cells were centrifuged again at 5,000 $\times$ g for 5 min at room temperature and resuspended to $\mathrm{OD}_{600} 10$ in nitrogen-depleted media. For nitrogen-depleted stationary phase cultures, the resuspended cells were used as the starting point. For growth phase cultures, the resuspended cells were inoculated into growth phase media at $\mathrm{OD}_{600} 0.03$. For the growth phase cultivations, mineral salt medium was used, containing $20 \mathrm{~g} / \mathrm{L}$ glucose, $5 \mathrm{~g} / \mathrm{L}$ ammonium sulfate, $12 \mathrm{~g} / \mathrm{L}$ potassium phosphate (pH 6.0), and $3.4 \mathrm{~g} / \mathrm{L} \mathrm{YNB} \mathrm{(w/o} \mathrm{amino} \mathrm{acids,} \mathrm{w/o} \mathrm{ammonium}$ sulfate). For the nitrogen-depleted stationary phase cultivations, the media contained $20 \mathrm{~g} / \mathrm{L}$ glucose, $12 \mathrm{~g} / \mathrm{L}$ potassium phosphate
(pH 6.0), and $3.4 \mathrm{~g} / \mathrm{L}$ YNB (w/o amino acids, w/o ammonium sulfate). For the fatty alcohol degradation test, cells were cultivated in triplicates in $12-\mathrm{ml}$ round bottom glass vials filled with $2-\mathrm{ml}$ media at $30^{\circ} \mathrm{C}$ at $250 \mathrm{RPM}$ in a MaxQ 8000 Orbital Shaker (Thermo Fisher). The cultivation medium contained 500 $\mathrm{mg} / \mathrm{L}$ hexadecanol, $500 \mathrm{mg} / \mathrm{L}$ octadecanol, $20 \mathrm{~g} / \mathrm{L}$ ethanol, $2 \mathrm{~g} / \mathrm{L}$ glucose, $1.7 \mathrm{~g} / \mathrm{L}$ YNB w/o amino acids and ammonium sulfate, 12 $\mathrm{g} / \mathrm{L}$ potassium phosphate ( $\mathrm{pH} 6.0$ ), and $5 \mathrm{~g} / \mathrm{L}$ ammonium sulfate. Biological triplicates were done for each strain and condition.

\section{HPLC Analysis}

Five hundred-microliter samples were taken at each sampling point, centrifuged in Eppendorf tubes at $5,000 \times \mathrm{g}$ at $4^{\circ} \mathrm{C}$ for $5 \mathrm{~min}$, and supernatants were stored at $-20^{\circ} \mathrm{C}$ until analysis. Supernatants were analyzed for the presence of ethanol, glucose, glycerol, and acetate using HPLC UltiMate 3000 (Thermo Fisher) with Aminex HPX87H ion exclusion column. Samples were run for $30 \mathrm{~min}$ at $0.600 \mathrm{ml} / \mathrm{min}$ at $60^{\circ} \mathrm{C}$ using $5 \mathrm{mM} \mathrm{H} 2 \mathrm{SO} 4$ as eluent. Compounds were detected using a Dionex RI101 and DAD-3000 detectors (Dionex) for RI and UV detection, respectively.

\section{Liquid Chromatography-Mass Spectrometry (LC-MS) Analysis}

For Y. lipolytica cultures, supernatant samples from section HPLC Analysis were also analyzed for the presence of tricarboxylic acid (TCA) cycle-derived organic acids (malic acid, succinic acid, citric acid, isocitric acid, pyruvic acid, aketoglutaric acid, and fumaric acid). LC-MS data were collected on the EVOQ Elite Triple Quadrupole Mass Spectrometer system coupled with an Advance UHPLC pump (Bruker, Fremont, CA). Samples were held in the CTC HTS PAL autosampler at a temperature of $5.0^{\circ} \mathrm{C}$ during the analysis. $1-\mu \mathrm{l}$ injections of the sample were made onto a Waters ACQUITY HSS T3 C18 UHPLC column, with a 1.8- $\mu \mathrm{m}$ particle size, $2.1 \mathrm{~mm}$ i.d., and $100 \mathrm{~mm}$ long. The column was maintained at $30.0^{\circ} \mathrm{C}$. The solvent system used was solvent $\mathrm{A}$ (MilliQ water with $0.1 \%$ formic acid) and solvent B (acetonitrile with $0.1 \%$ formic acid). The flow rate was $0.400 \mathrm{ml} / \mathrm{min}$ with an initial solvent composition of $\% \mathrm{~A}=100$ and $\% \mathrm{~B}=0$ held until $0.50 \mathrm{~min}$; the solvent composition was then changed following a linear gradient until it reached $\% \mathrm{~A}=5.0$ and $\% \mathrm{~B}=95.0$ at 1.00 $\mathrm{min}$. This was held until $1.79 \mathrm{~min}$ when the solvent was returned to the initial conditions, and the column was re-equilibrated until $4.00 \mathrm{~min}$. The column eluent flowed directly into the heated ESI probe of the MS, which was held at $250^{\circ} \mathrm{C}$ and a voltage of 2,500 V. MRM data was collected in negative ion mode; the target masses are shown in Supplementary Table S2. The other MS settings were as follows: sheath gas flow rate of 50 units, nebulizer gas flow rate of 50 units, cone gas flow rate of 20 units, cone temp was $350^{\circ} \mathrm{C}$, and collision gas pressure $1 \mathrm{~m}$ Torr.

\section{Cell Dry Weight}

The cell dry weight (CDW) was determined by pre-weighing dried $0.45-\mu \mathrm{m}$ cellulose nitrate membrane filters (VWR), filtering $10-\mathrm{ml}$ culture across the membrane, washing with 10 $\mathrm{ml}$ water, drying at $60^{\circ} \mathrm{C}$, and weighing the dried filter with 
biomass. The OD/CDW ratio was determined to be constant for all the strains throughout all the cultivations, and for the cultures throughout this study, the biomass was estimated by measuring the $\mathrm{OD}_{600}$ using a NanoPhotometer Pearl (Implen) and calculating the biomass dry weight using conversion factors of $0.12(\mathrm{~g} / \mathrm{L}) / \mathrm{OD}$ or $0.14(\mathrm{~g} / \mathrm{L}) / \mathrm{OD}$ for $S$. cerevisiae and Y. lipolytica, respectively.

\section{Fatty Alcohol Extraction}

Fatty alcohols were analyzed by collecting $1 \mathrm{ml}$ of culture broth into a 4-ml glass vial and adding $10-\mu \mathrm{l}$ internal standard $(2 \mathrm{~g} / \mathrm{L}$ of methyl cis-10-heptadecanoate dissolved in $100 \%$ ethanol). Samples were vortexed for $3 \mathrm{~s}$ and frozen at $80^{\circ} \mathrm{C}$ until further processing. To perform the extraction, samples were freeze-dried for $2-3$ days at $-30^{\circ} \mathrm{C}$ under vacuum in a freeze-drying system (Labconco). $1 \mathrm{ml} \mathrm{2:1} \mathrm{chloroform:methanol} \mathrm{mixture} \mathrm{was} \mathrm{added}$ to each freeze-dried sample to disrupt the cells, vortexed for 15 min in a DVX-2500 multi-tube vortexer (VWR), and left at room temperature for $4 \mathrm{~h}$. The solvents were subsequently evaporated under a nitrogen stream. $1 \mathrm{ml}$ hexane was added to the sample vials, vortexed for $15 \mathrm{~min}$ in a DVX-2500 multi-tube vortexer (VWR), and incubated at room temperature overnight. Samples were transferred to new vials and stored at $-20^{\circ} \mathrm{C}$ until analyzed.

The analysis was carried out on a GC-MS using an INNOWax column $(30 \mathrm{~m} \times 0.25 \mathrm{~mm} \times 0.25 \mu \mathrm{m})$ with helium as carrier gas. The injector was set to splitless mode at $250^{\circ} \mathrm{C}$; the oven temperature was set to $80^{\circ} \mathrm{C}$ for $1 \mathrm{~min}$, increased at a rate of $10^{\circ} \mathrm{C} /$ min to $210^{\circ} \mathrm{C}$, followed by a hold at $210^{\circ} \mathrm{C}$ for $15 \mathrm{~min}$, increased at a rate of $10^{\circ} \mathrm{C} / \mathrm{min}$ to $230^{\circ} \mathrm{C}$ followed by a hold at $230^{\circ} \mathrm{C}$ for 20 min. The GC-MS was operated in electron impact mode $(70 \mathrm{eV})$, scanning at the range $30-400 \mathrm{~m} / \mathrm{z}$. Compounds were quantified relative to the internal standard (methyl cis-10-heptadecenoate).

\section{Fatty Alcohol Degradation}

Fatty alcohol degradation was estimated by cultivating cells in media containing fatty alcohols. Strains used were CEN. PK113-7D (S. cerevisiae reference), ST6849 (S. cerevisiae pex104, hfd1A), W29 (Y. lipolytica reference), and ST6770 (Y. lipolytica pex $10 \Delta, f a o 1 \Delta, h f d 1 \Delta, h f d 4 \Delta$ ) (Table 1). The negative controls did not contain any cells. Cultures were inoculated to a starting $\mathrm{OD}_{600}$ of 1.0 and incubated for $96 \mathrm{~h}$. The whole cultivation tube containing $2 \mathrm{ml}$ culture was taken as sample and processed for fatty alcohol quantification according to the previous description
(Cell Dry Weight), but with double volumes to account for the increased sample size. The detection of slightly lower levels of hexadecanol and slightly higher levels of octadecanol than expected is likely due to an experimental error, which is rather high when working with hydrophobic substances in small volumes. Upon addition of fatty alcohols to the media, they form a sticky floating white precipitate and, while we tried to recover the whole remaining fatty alcohol by adding organic solvent directly to the tube, some of it may still remain on the walls of the tube.

\section{RNA Sequencing and Gene Expression Analysis}

Growth phase cultures were sampled at OD 2-3 ( 6 generations), when the cultures were in the mid-exponential growth phase and the unlabeled biomass from inoculum was diluted to less than $3 \%$. Nitrogen-depleted cultures were sampled when ca. $50 \%$ of glucose was consumed, which was after $12 \mathrm{~h}$ for S. cerevisiae and after $48 \mathrm{~h}$ for $Y$. lipolytica. The sample volume corresponding to $5 \times 10^{7}-10^{8}$ cells was added into $50-\mathrm{ml}$ Falcon tube, filled with ice. The tubes were centrifuged at $4^{\circ} \mathrm{C}$ for $1 \mathrm{~min}$, the liquid was discarded, and the pellet was snap frozen in liquid nitrogen and stored at $-80^{\circ} \mathrm{C}$ until further processing. The cell lysis was carried out in 2 ml-screw cap tubes with $600-\mu$ l RLT buffer and 500- $\mu$ l glass beads using a Precellys 24 at 6000 RPM for $4 \times 25$ $\mathrm{s}$, with $60 \mathrm{~s}$ on ice in between. RNA was subsequently extracted using the RNeasy kit (Qiagen), according to the manufacturer's instructions.

Library preparation was carried out using the TruSeq Stranded mRNA Library Prep Kit (Illumina), and the TruSeq RNA CD indexes (Illumina). Sequencing was carried out using a NextSeq 500 system (Illumina), with NextSeq Mid and High Output v2 Kits (150 cycles), as 75 bp paired-end reads. Index (i7 and i5) reads: 8 bp, flow cell loading: $1.08 \mathrm{pM}$, sequencing chemistry: 2-channel sequencing-by-synthesis (SBS) technology. PhiX was added at $2.5 \%$. Sequencing facility: NGS lab at the Novo Nordisk Foundation Center for Biosustainability.

The RNA-seq data was processed using KBase (Arkin et al., 2018), and unless specified, default settings were used. Reads were trimmed using Trimmomatic v0.36 (post-tail crop length: 73, head crop length: 14) (Bolger et al., 2014). Read quality was assessed by FastQC. Reads were merged using Multiple ReadsLibs to One ReadsLib v1.0.1. Reads were aligned to the

TABLE 1 | Strains used in this study.

\begin{tabular}{|c|c|c|c|}
\hline Name & ID & Genotype & Reference \\
\hline S. cerevisiae reference & CEN.PK113-7D & MATa & Entian and Kötter, 2007 \\
\hline S. cerevisiae non-producer & ST6849 & pex10 $\Delta$, hfd1 $\Delta$, MATa & This study \\
\hline S. cerevisiae fatty alcohol producer & ST6989 & $4 \times$ MalFAR, pex $10 \Delta$, hfd1 $1 \Delta$, MATa & This study \\
\hline Y. lipolytica reference & W29 & MATa & Gaillardin et al., 1973 \\
\hline Y. lipolytica non-producer & ST6770 & 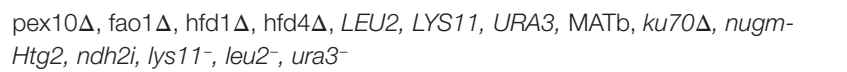 & This study \\
\hline Y. lipolytica fatty alcohol producer & ST6987 & 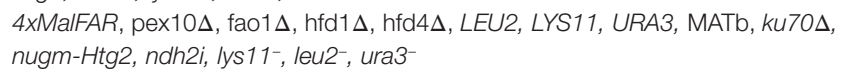 & This study \\
\hline
\end{tabular}


reference genome using HISAT2 v2.1.0 (Kim et al., 2015). The reference genomes used were modified versions of S288C and W29 (Clib89) for S. cerevisiae and Y. lipolytica, respectively; the modification consisted of the addition of the expressed malFAR genes. Alignment quality was assessed using Qualimap2 v2.2.1 (Okonechnikov et al., 2016). Alignments were assembled using StringTie v1.3.3b (not allowing for novel transcripts) (Pertea et al., 2015). The analysis was carried out separately using EdgeR v3.24.3 (Robinson et al., 2010) in R v3.5.1, using TMM normalization as well as false discovery rate (FDR) correction using the Benjamini-Hochberg method. EdgeR was used to calculate the effect of either nitrogen-depletion or fatty alcohol production in both species. Contrasts were set as " $(\mathrm{A}+\mathrm{B}) / 2$ $(\mathrm{C}+\mathrm{D}) / 2$ " where $\mathrm{A}$ and $\mathrm{B}$ belonged to the same condition (e.g., growth phase), and $\mathrm{C}$ and $\mathrm{D}$ belonged to the same condition (e.g., nitrogen depletion) An exception was made for the effect of fatty alcohol production in Y. lipolytica, where only genes differentially expressed between strains in nitrogen-depleted conditions were considered, due to a lack of difference between the strains during growth phase. Differentially expressed genes predicted by EdgeR were filtered for a $p$-value or $0.01, \log 2 \mathrm{CPM}$ of 1 , and $\log 2$-fold change of 2 .

GO term enrichment analysis was carried out by the PANTHER v14.0-based online tool (Ashburner et al., 2000; Thomas et al., 2003; Mi et al., 2017; The Gene Ontology Consortium, 2017) available at www.geneontology.org. Enrichment analysis was carried out for biological process GO terms using Fisher's exact test and Bonferroni correction. Results were filtered for a p-value less than 0.05 and fold enrichment greater than 2.5.

RNA-seq data has been uploaded to European Nucleotide Archive (PRJEB32352). A list of differentially expressed genes is attached as Supplementary File S3.

\section{${ }^{13} \mathrm{C}$-Metabolic Flux Analysis}

Cells were cultivated as previously described (Cultivation), with the exception that labeled glucose was used. One replicate was made with $20 \% \mathrm{U}-{ }^{13} \mathrm{C}$ glucose (99\% purity, Euriso-Top $\mathrm{GmbH}$, Saarbrücken, Germany) and $80 \%$ non-labeled glucose. Two replicates were made with $20 \% \mathrm{U}-{ }^{13} \mathrm{C}$ glucose and $80 \% 1-{ }^{13} \mathrm{C}$ glucose (99\% purity, Euriso-Top $\mathrm{GmbH}$, Saarbrücken, Germany). The two isotopomers $\left(1-{ }^{13} \mathrm{C}\right.$ glucose and $\mathrm{U}-{ }^{13} \mathrm{C}$ glucose $)$ are commonly used in ${ }^{13} \mathrm{Cmetabolic}$ flux analysis as they provide a good flux resolution at a reasonable cost. Furthermore, the single replicate of $20 \% \mathrm{U}^{13} \mathrm{C}$ glucose can be used to estimate the quality of the MS data. The labeling strategy was informed by previous publication (Zamboni et al., 2009). A cell amount of $0.3 \mathrm{mg}$ CDW were harvested at OD 2 ( $\sim 6$ generations) and washed with $1 \mathrm{ml}$ cold $\mathrm{NaCl}(0.9 \%)$. The pellet was stored at $-80^{\circ} \mathrm{C}$ until processing. The samples were resuspended in $150 \mu \mathrm{l} \mathrm{HCl}(6 \mathrm{M})$, transferred to a glass vial, and incubated at $105^{\circ} \mathrm{C}$ for $6 \mathrm{~h}$ to hydrolyze the cell pellet as previously described (Schmitz et al., 2017). Samples were dried until only a dark brown residue remained by heating the open vial at $80^{\circ} \mathrm{C}$ under a fume hood. The residue was resuspended in $30 \mu \mathrm{l}$ acetonitrile. Samples were derivatized by the addition of MBDSTFA at a $1: 1$ ratio and incubated at $85^{\circ} \mathrm{C}$ for $1 \mathrm{~h}$. Samples were analyzed by GC-MS according to a previously described protocol (Kildegaard et al., 2016). Raw GC-MS data was corrected using iMS2Flux v.7.2.1 (Poskar et al., 2012). Fluxes were calculated using parameter continuation in the INCA v1.7 software (Young, 2014). The model used was adapted from previous publication (Wasylenko and Stephanolous, 2015), see Supplementary File S4 for the final model used, as well as for the full set of calculated fluxes.

\section{Intracellular Metabolome Analysis}

Samples corresponding to approximately $0.25 \mathrm{mg}$ CDW were taken for each replicate. For growth phase samples, samples were taken at mid-growth phase (OD 1-2, 6 generations). For the nitrogen-depletion samples, samples were taken at 12 or $48 \mathrm{~h}$, respectively, for S. cerevisiae and Y. lipolytica strains. The sampling method used was adapted from a previous publication (McCloskey et al., 2015b), with the addition of an extra extraction step, in which the filter with the quenched biomass was transferred into a 50-ml Falcon tube containing $5 \mathrm{ml}$ boiling ethanol, together with the $1 \mathrm{ml}$ extraction solvent used for quenching (containing internal standard). The filter was incubated at $80^{\circ} \mathrm{C}$ in the boiling ethanol for $90 \mathrm{~s}$, the tube was quickly vortexed, the filter was flipped, and the tube was incubated for another $90 \mathrm{~s}$. The solution was aliquoted into 2-ml Eppendorf tubes and processed according to the previously described protocol. See Supplementary File S5 for full details. The analysis was conducted according to previous publication (McCloskey et al., 2015a).

\section{RESULTS}

\section{Establishing Fatty Alcohol Production in S. cerevisiae and Y. lipolytica}

The first step towards creating fatty alcohol-producing yeast strains was to reduce the degradation of fatty alcohols as described in Borodina et al. (2018). In S. cerevisiae, we chose to delete the genes encoding peroxisomal biogenesis factor Pex $10 \mathrm{p}$ and aldehyde dehydrogenase Hfd1p (Figure 2). The deletion of PEX10 prevents the formation of peroxisomes, where $\beta$-oxidation of fatty acids occurs. The HFD1 gene was shown in a previous study (Buijs et al., 2015) to be responsible for the degradation of fatty alcohols in S. cerevisiae. In Y. lipolytica, we deleted PEX10 and two out of four aldehyde dehydrogenase-coding genes HFD1 and HFD4, the ones that were previously reported to have the highest activity (Iwama et al., 2014). Additionally, we deleted the FAO1 gene encoding a fatty alcohol oxidase in Y. lipolytica (Figure 2).

In order to investigate if the chosen gene knockouts reduced the degradation of fatty alcohols, we cultivated the nonengineered and engineered strains in the medium supplemented with approximately $0.5 \mathrm{~g} / \mathrm{L}$ each of hexadecanol and octadecanol for $96 \mathrm{~h}$ and analyzed the remaining fatty alcohol concentration (Figure 3A). There was no significant difference between the final concentrations of fatty alcohols between the cultures of $S$. cerevisiae strains and the control experiment without cell addition. As it has been shown previously that $S$. cerevisiae can 


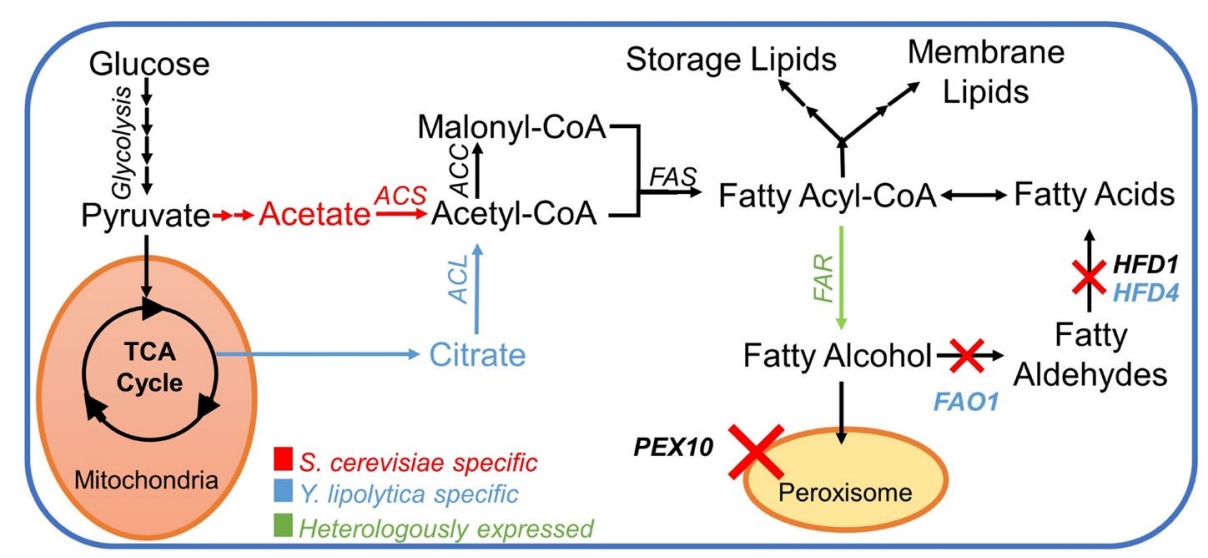

FIGURE 2 | Pathway for fatty alcohol production; a simplified pathway for the metabolic conversion of glucose into fatty alcohols in S. cerevisiae and Y. lipolytica. Color-coded text and arrows: red, pathway in Saccharomyces cerevisiae; blue, pathway in Yarrowia lipolytica; green, heterologous reactions. The red X-symbol signifies corresponding gene knockouts. Abbreviations: ACL, ATP citrate lyase; ACS, acetyl-CoA synthase; ACC, acetylCoA carboxylase; FAS, fatty acid synthase complex; FAR, fatty acyl-CoA reductase; PEX10, peroxin 10; FAO1, fatty alcohol oxidase; HFD1, fatty aldehyde dehydrogenase 1 (ALDH1); HFD4, fatty aldehyde dehydrogenase $4(A L D H 4)$.

A

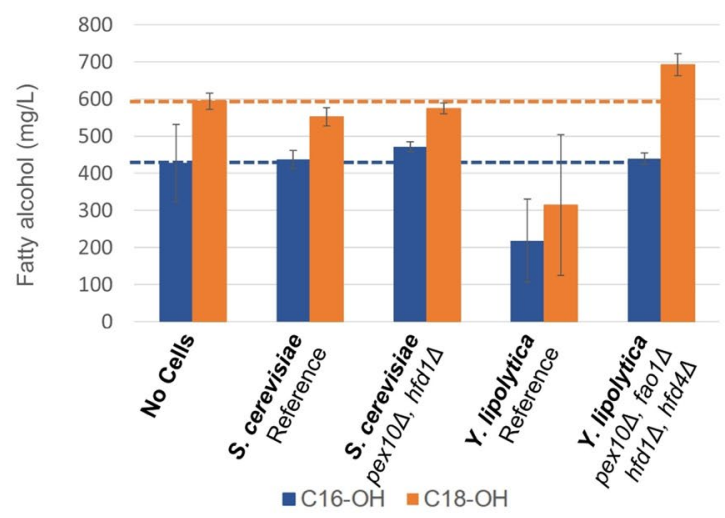

B

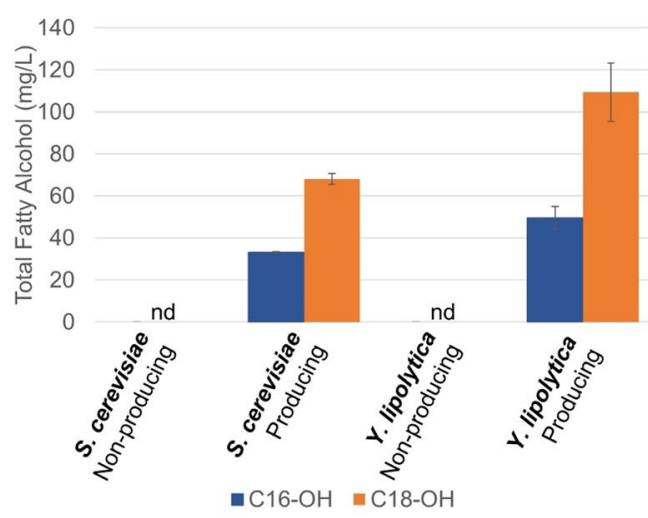

FIGURE 3 | Fatty alcohol degradation and production (A) Fatty alcohol degradation was estimated by cultivating yeast strains in the presence of extracellular fatty alcohol mixture consisting of hexadecanol $(\mathrm{C} 16-\mathrm{OH})$ and octadecanol $(\mathrm{C} 18-\mathrm{OH})$ and quantifying the residual concentrations. (B) Production of fatty alcohols by engineered strains of S. cerevisiae and Y. lipolytica on minimal medium after $96 \mathrm{~h}$, nd: not detected. Data shown are mean values \pm standard deviations of biological triplicates.

degrade fatty alcohols (d'Espaux et al., 2017) that it produces, the lack of apparent degradation of extracellular fatty alcohols could be explained by poor uptake. As for Y. lipolytica, the reference strain degraded approximately half of the added hexadecanol and octadecanol, whereas the engineered strain (pex10 $\Delta$, fao $1 \Delta$, $h f d 1 \Delta, h f d 4 \Delta$ ) showed no fatty alcohol degradation, indicating that the knockouts had impaired the ability of the strain to degrade fatty alcohols, as intended.

In the next step, we integrated four copies of the malFAR fatty acyl-CoA reductase (FAR) gene into the yeast strains with reduced fatty alcohol degradation. The FAR genes were expressed from strong constitutive promoters, TEF1 and TDH3 (GPD) for S. cerevisiae and from TEFintron (Tai and Stephanopoulos, 2013) and GPD promoters for Y. lipolytica. The strong constitutive promoters were selected to ensure that fatty alcohol biosynthetic genes were expressed both in the growth phase and in the nitrogen-depletion phases. The fatty alcohol production was evaluated in small-scale shake flask cultivations in mineral media for $96 \mathrm{~h}$ (Figure 3B). The $S$. cerevisiae strain carrying the FAR produced $105 \pm 3 \mathrm{mg} / \mathrm{L}$ of total fatty alcohols, whereas the $Y$. lipolytica strain produced $166 \pm 20 \mathrm{mg} / \mathrm{L}$ of total fatty alcohols. These titers are similar to the shake flasks titers reported by previous study (Xu et al., 2016). Xu et al. subsequently reported $2.2 \mathrm{~g} / \mathrm{L}$ in bioreactors using the same strains, the highest reported levels on minimal media.

Throughout this study, non-producing strains of $S$. cerevisiae

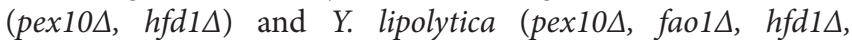
$h f d 4 \Delta$ ) were compared with fatty alcohol-producing strains

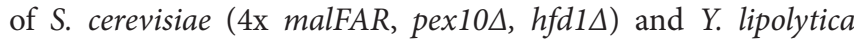

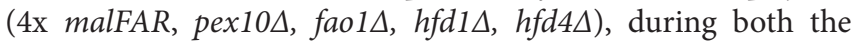


exponential growth phase as well as during a nitrogen-depleted stationary phase (Table 1). Pre-cultures were grown on minimal media until the late exponential phase, and subsequently washed and resuspended in fresh minimal media for growth or in nitrogendepletion media for stationary phase studies. This experimental set-up allowed for simplified parallel investigation of each phase individually, which could be made to occur in sequence as a result of nitrogen consumption in an industrial setting. The nitrogendepleted stationary phase is of interest since it has previously been shown to increase the flux via fatty acyl-CoA to triacylglycerides and lipid accumulation (Pomraning et al., 2016).

The fatty alcohol-producing strain of S. cerevisiae had a $50 \%$ lower maximum specific growth rate, $\mu_{\max }$, and reached a lower final OD, $\sim 40 \%$ of the final OD reached by the parental strain not expressing FAR genes (Figure 4A and Table 2). Although no time series data is available beyond the $50 \mathrm{~h}$, we made some separate experiments under the same conditions, and no further increase in OD was observed between 50 and $96 \mathrm{~h}$ for the producing strain. In contrast, the $\mu_{\max }$ of $Y$. lipolytica was not affected by expression of fatty alcohol reductase genes, and the strain with fatty alcohol production grew to a 2.6-fold higher final OD (Figure 4B and Table 2). When the strains were transferred to a medium without nitrogen and cultivated for $21 \mathrm{~h}$, the OD of the non-producing $S$. cerevisiae strain increased 1.9-fold (Figure 4C). The OD of the non-producing $Y$. lipolytica strain decreased slightly, indicating some negative effect of the introduced gene deletions (Figure 4D).

Glucose uptake rates varied greatly between the hosts and conditions. S. cerevisiae exhibited a $\sim 13$-fold and $\sim 5$-fold higher glucose uptake rate in growth phase and nitrogen-depleted stationary phase, respectively, compared to Y. lipolytica. Furthermore, the uptake rate was $\sim 17$-fold higher and $\sim 6$-fold higher in the growth phase than in the nitrogen-depletion phase in S. cerevisiae and $Y$. lipolytica, respectively (Table 2).

As for by-products, S. cerevisiae primarily secreted ethanol, as well as some acetate and glycerol. There was no major change in the by-product secretion of the producing and non-producing strains of S. cerevisiae. Y. lipolytica secreted several TCA cycleassociated organic acids, primarily pyruvate, $\alpha$-ketoglutarate, citrate, and malate. The fatty alcohol-producing strain of $Y$. lipolytica exhibited a 2-3-fold lower by-product secretion rates for all the measured metabolites, during both exponential growth and nitrogen-depleted stationary phase, plausibly due to a redirection of the flux toward fatty alcohols. The relative secretion rate (relative to glucose uptake rate) of isocitrate in the producing $Y$. lipolytica strain was reduced by $\sim 20$-fold in the nitrogen-depleted condition. The reduced isocitrate secretion rate could potentially be explained by an increased transport of mitochondrial citrate to the cytoplasm, followed by conversion of citrate to acetyl-CoA (catalyzed by ATP-citrate lyase), which in turn would be used for fatty alcohol production. In response to nitrogen-depletion, $Y$. lipolytica exhibited a 5-25-fold relative increase in secretion rate of all by-products analyzed, except for citrate, which was secreted with a $\sim 200$-fold increased relative rate. During nitrogendepletion, $Y$. lipolytica secreted $\sim 50 \%$ and $\sim 25 \%$ of the total carbon consumed, in the form of organic acid by-products for the non-producing and producing strain, respectively. Additionally, the fatty alcohol-producing strain produced fatty alcohols corresponding to $\sim 8 \%$ of the carbon consumed. Both S. cerevisiae and Y. lipolytica showed detectable fatty alcohol production only in nitrogen-depleted conditions (Table 2).

\section{A}
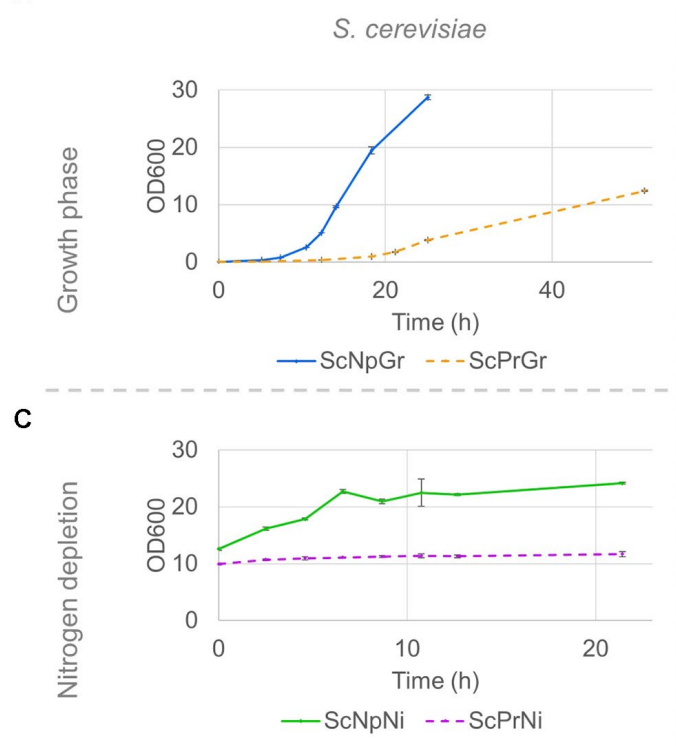

B

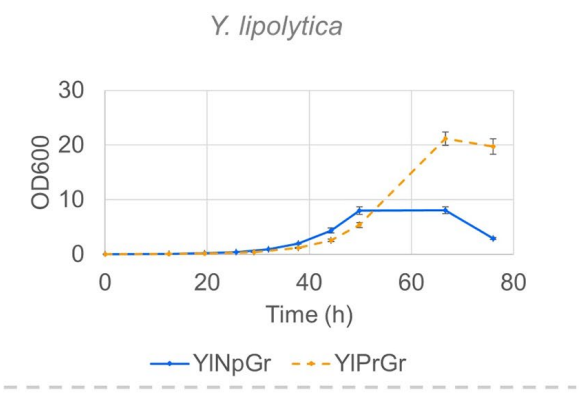

D

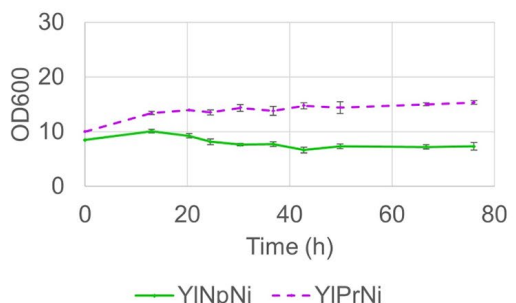

FIGURE 4 | Strain growth in minimal media and nitrogen-depleted media; Growth curves of S. cerevisiae (A, C) and Y. lipolytica strains (B, D) on minimal media (A, B) and nitrogen-depletion media (C, D). Pre-cultures were grown on minimal media until the late exponential phase, and subsequently washed and resuspended in either fresh minimal media or in nitrogen-depletion media. Data shown are mean values \pm standard deviations of biological triplicates. Sc, Saccharomyces cerevisiae; YI, Yarrowia lipolytica, Pr, fatty alcohol producing; Np, non-producing; Gr, growth phase; Ni, nitrogen-depleted stationary phase. 
TABLE 2 | Uptake-, secretion-, and growth rate. Sc, Saccharomyces cerevisiae; YI, Yarrowia lipolytica, Pr, fatty alcohol producing; Np, non-producing; Gr, growth phase; Ni, nitrogen-depleted stationary phase; nd, not determined. Data shown are mean values \pm standard deviations of biological triplicates.

\begin{tabular}{|c|c|c|c|c|c|c|c|}
\hline & Glucose & Ethanol & Acetate & Glycerol & Succinate & Malate & Isocitrate \\
\hline & $\mathrm{mmol} \mathrm{g}^{-1} \mathrm{~h}^{-1}$ & $\mathrm{mmol} \mathrm{g}{ }^{-1} \mathrm{~h}^{-1}$ & $\mathrm{mmol} \mathrm{g}{ }^{-1} \mathrm{~h}^{-1}$ & $\mathrm{mmol} \mathrm{g}{ }^{-1} \mathrm{~h}^{-1}$ & $\mu \mathrm{mol} \mathrm{g} \mathrm{g}^{-1} \mathrm{~h}^{-1}$ & $\mu \mathrm{mol} \mathrm{g} \mathrm{g}^{-1} \mathrm{~h}^{-1}$ & $\mu \mathrm{mol} \mathrm{g} \mathrm{g}^{-1} \mathrm{~h}^{-1}$ \\
\hline ScNpGr & $15.7 \pm 0.29$ & 22. \pm 0.3 & $1.41 \pm 0.1$ & $2.81 \pm 0.0$ & nd & nd & nd \\
\hline ScPrGr & $15.4 \pm 0.26$ & $20.6 \pm 0.4$ & $2.3 \pm 0.3$ & $3.89 \pm 0.3$ & nd & nd & nd \\
\hline ScNpNi & $1.17 \pm 0.12$ & $0.9 \pm 0.5$ & $0.2 \pm 0.0$ & $0.0 \pm 0.0$ & nd & nd & nd \\
\hline ScPrNi & $1.61 \pm 0.10$ & $2.4 \pm 0.9$ & $0.1 \pm 0.0$ & $0.3 \pm 0.0$ & nd & nd & nd \\
\hline YINpGr & $1.52 \pm 0.19$ & nd & nd & nd & $1.2 \pm 0.4$ & $8.3 \pm 1.9$ & $1.1 \pm 0.1$ \\
\hline YIPrGr & $2.04 \pm 0.20$ & nd & nd & nd & $0.8 \pm 0.0$ & $5.5 \pm 0.2$ & $0.4 \pm 0.0$ \\
\hline YINpNi & $0.35 \pm 0.01$ & nd & nd & nd & $5.0 \pm 0.7$ & $31.3 \pm 3.8$ & $6.6 \pm 1.2$ \\
\hline \multirow[t]{2}{*}{ YIPrNi } & $0.22 \pm 0.03$ & nd & nd & nd & $1.8 \pm 0.3$ & $9.1 \pm 0.6$ & $0.2 \pm 0.5$ \\
\hline & $\begin{array}{l}\text { Citrate } \\
\mu \mathrm{mol} \mathrm{g}{ }^{-1} \mathrm{~h}^{-1}\end{array}$ & $\begin{array}{l}\boldsymbol{\alpha} \text {-Ketoglutarate } \\
\mu \mathrm{mol} \mathrm{g} \mathrm{g}^{-1} \mathrm{~h}^{-1}\end{array}$ & $\begin{array}{l}\text { Fumarate } \\
\mu \mathrm{mol} \mathrm{g}{ }^{-1} \mathrm{~h}^{-1}\end{array}$ & $\begin{array}{l}\text { Pyruvate } \\
\mu \mathrm{mol} \mathrm{g}-1 \mathrm{~h}^{-1}\end{array}$ & $\begin{array}{l}\text { Fatty alcohols } \\
\mu \mathrm{mol} \mathrm{g}{ }^{-1} \mathrm{~h}^{-1}\end{array}$ & $\begin{array}{l}\text { Growth rate } \\
\mathrm{h}^{-1}\end{array}$ & \\
\hline ScNpGr & nd & nd & nd & nd & nd & $0.33 \pm 0.00$ & \\
\hline ScPrGr & nd & nd & nd & nd & nd & $0.17 \pm 0.00$ & \\
\hline $\mathrm{ScNpNi}$ & nd & nd & nd & nd & nd & nd & \\
\hline $\mathrm{ScPrNi}$ & nd & nd & nd & nd & $1.9 \pm 0.7$ & nd & \\
\hline YINpGr & $1.1 \pm 0.8$ & $30.2 \pm 3.9$ & $1.7 \pm 0.4$ & $79.7 \pm 8.9$ & nd & $0.13 \pm 0.00$ & \\
\hline YIPrGr & $0.6 \pm 0.1$ & $11.9 \pm 0.9$ & $1.0 \pm 0.1$ & $33.2 \pm 5.0$ & nd & $0.12 \pm 0.00$ & \\
\hline YINpNi & $54.9 \pm 3.2$ & $39.1 \pm 13.4$ & $7.2 \pm 1.4$ & $102.7 \pm 5.4$ & nd & nd & \\
\hline YIPrNi & $12.6 \pm 1.2$ & $19.9 \pm 2.2$ & $1.3 \pm 0.1$ & $29.9 \pm 2.7$ & $6.0 \pm 0.9$ & nd & \\
\hline
\end{tabular}

\section{${ }^{13} \mathrm{C}$-Metabolic Flux Analysis}

In order to get insight into the strains' response to fatty alcohol production on a metabolic level, ${ }^{13} \mathrm{C}$-fluxomics and targeted metabolomics analyses were conducted. The flux analysis determines the fluxes (conversion rates) between metabolites. Fluxes reveal the flow of carbon through the cell and the distribution of carbon flux between alternative pathways. For ${ }^{13} \mathrm{C}$-flux analysis, the cells were cultivated on a mix of labeled and unlabeled glucose. The incorporation of labeled carbons $\left({ }^{13} \mathrm{C}\right)$ into proteinogenic amino acids was measured by GC-MS. The ${ }^{13} \mathrm{C}$-flux analysis method requires a metabolic steady state and, if based on measurements of proteinogenic amino acids, growing cells. Therefore, the fluxes were only estimated for the exponential growth phase, which can be considered to represent a quasi-steady-state condition.

The central carbon flux distributions in S. cerevisiae and Y. lipolytica were very different (Figure 5). The S. cerevisiae strains processed approximately $90 \%$ of the carbon from glucose through glycolysis, whereas only around 10\% of the carbon went into the pentose phosphate pathway. The cells channeled $\sim 50 \%$ of the carbon from glucose to ethanol, primarily relying on the energy generated in the fermentation process. $Y$. lipolytica is a non-fermenting yeast, so the energy is generated by oxidative phosphorylation in the mitochondria. Besides the higher TCA cycle flux associated with this respiratory lifestyle, Y. lipolytica differed from S. cerevisiae by diverting nearly half of the internalized glucose into the pentose phosphate pathway generating substantial amounts of NADPH. NADPH is a key redox co-factor for fatty acid and fatty alcohol biosynthesis. Additionally, Y. lipolytica had a $\sim 5$-fold higher relative flux (relative to glucose uptake) towards cytosolic acetyl-CoA than S. cerevisiae. Acetyl-CoA is the precursor for fatty acyl-CoA, which in turn is the precursor for fatty alcohols, and other fatty acid-derived compounds. It is worth noting that S. cerevisiae and
Y. lipolytica utilize different pathways for the synthesis of acetylCoA from glucose (Figure 2). In S. cerevisiae, most of cytosolic acetyl-CoA is produced by the action of pyruvate decarboxylase, aldehyde dehydrogenase, and acetyl-CoA synthase. The NADPdependent ALD6 has previously been shown to contribute $40 \%$ of the NADPH generated in S. cerevisiae, with the remaining $60 \%$ being generated via the pentose phosphate pathway (Blank et al., 2005). In contrast, Y. lipolytica uses ATP-citrate lyase $(A C L)$ to make acetyl-CoA from citrate, which is exported from mitochondria with a simultaneous import of malate. The by-product of the ACL reaction is oxaloacetate, which is converted into malate. In Y. lipolytica, the NADPH is primarily generated through the pentose phosphate pathway (Wasylenko et al. 2015).

\section{Metabolomics}

Metabolomics analysis determines the intracellular metabolite concentrations. Metabolite pools can help to reveal limitations in precursor availability, as well as potentially limiting steps in the production pathway. Samples for metabolome analysis were taken during growth or nitrogen-depletion in producing and non-producing strains of both S. cerevisiae and Y. lipolytica and were rapidly filtered and quenched.

The metabolomics data revealed a decreased abundance of acetyl-CoA during nitrogen-depletion in both species, $\sim 3$ - and $\sim 6.5$-fold lower in S. cerevisiae and Y. lipolytica, respectively (Figure 5). This might indicate that fatty alcohol-producing strains encounter a limited precursor supply under these conditions. Y. lipolytica had increased levels of intermediates of the pentose phosphate pathway, which is consistent with the high flux through this pathway. Ribose 5-phosphate (r5p), ribulose 5-phosphate (ru5p), and sedoheptulose 7-phosphate (s7p) had a $\sim 3$-fold higher abundance in Y. lipolytica compared to S. cerevisiae strain. Given that this difference was observed with 


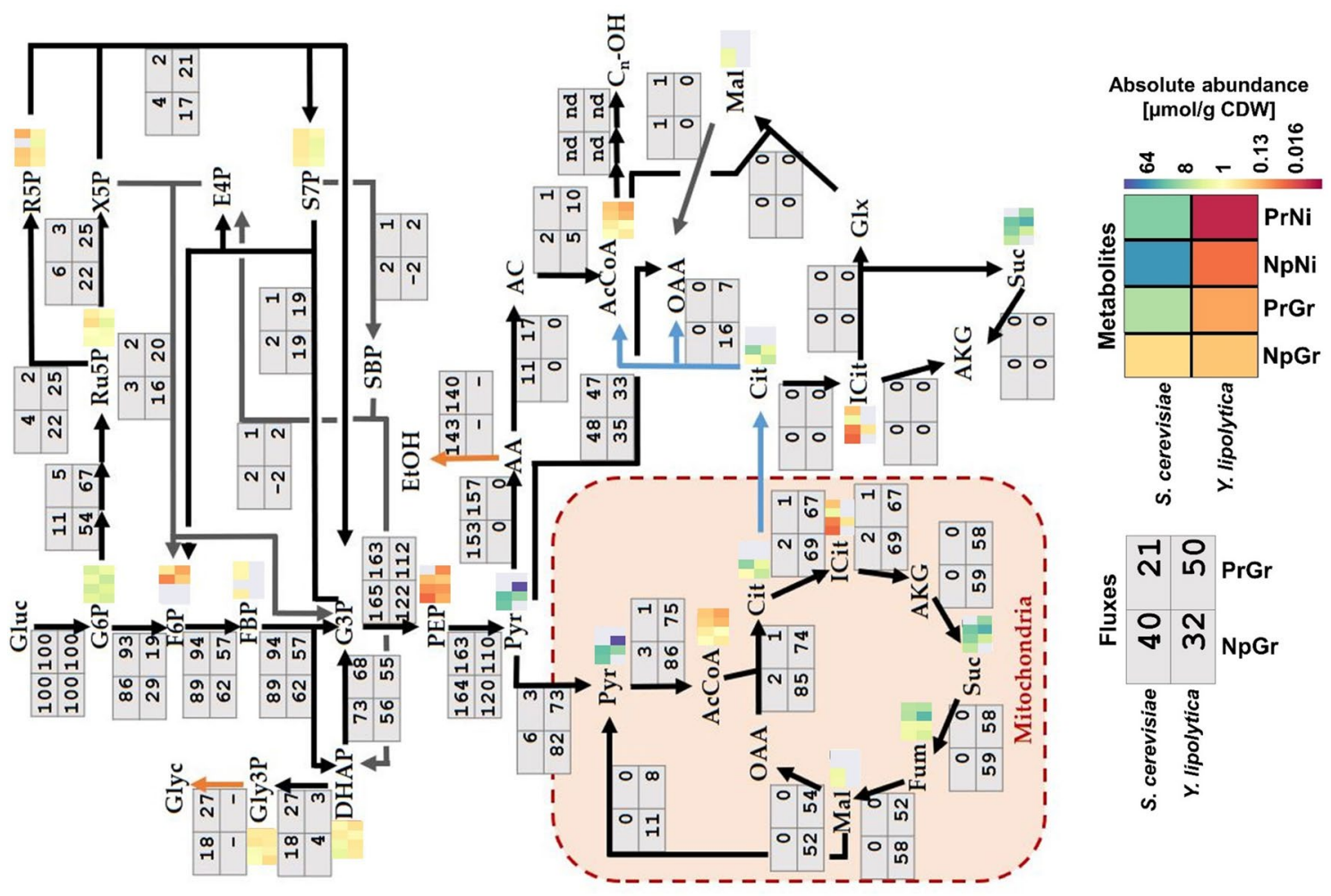

FIGURE 5 | Fluxes and metabolite levels in central carbon metabolism. Fluxes (gray boxes) are relative values, defined as the percentage of glucose uptake rate. Negative values indicate that the reaction is taking place in the opposite direction to what is indicated by the arrow. Metabolites (heatmaps) correspond to absolute abundances in $\mu \mathrm{mol} / \mathrm{g}$ CDW; gray means data missing (below the detection limit, saturated signal, or too high background in control sample). Sc: Saccharomyces cerevisiae, Yl: Yarrowia lipolytica, Pr: fatty alcohol producing, Np: non-producing, Gr: growth phase, Ni: nitrogen-depleted stationary phase.

both producing and non-producing strains, it appears to be an inherent feature of Y. lipolytica metabolism.

\section{Transcriptomics}

The gene expression profiles of fatty alcohol producing and non-producing strains were analyzed during growth and nitrogen-depletion via RNA sequencing. Principal component analysis (PCA) plots of the data revealed four separate sample groups in $S$. cerevisiae and three separate groups in Y. lipolytica (Figure 6). In S. cerevisiae, these groups represented the four different conditions (fatty alcohol-producing strain in growth and nitrogen-depletion, and non-producing strain in growth and nitrogen-depletion), indicating clear differences between all analyzed strains/conditions. In contrast, for Y. lipolytica, the three groups revealed that the producing and non-producing strains in the nitrogen-depletion phase were clearly different from each other and from the strains in the growth phase. But producing and non-producing strains in the growth phase were similar. Furthermore, direct comparison of the differentially expressed genes between the two conditions revealed only nine functionally annotated genes, out of which only four were also differentially expressed between the same two strains in nitrogendepleted conditions. Considering this similarity, only Y. lipolytica strains subjected to the nitrogen-depleted conditions were used to identify the differentially expressed genes in response to fatty alcohol production in Y. lipolytica. In both species, PC1 separates growth phase from nitrogen-depleted stationary phase and explains $59 \%$ and $47 \%$ of the difference in S. cerevisiae and $Y$. lipolytica, respectively.

In response to nitrogen-depletion (nitrogen-depleted stationary phase $v$ s. growth phase), S. cerevisiae differentially expressed 716 genes, 401 of which were upregulated in nitrogendepleted stationary phase, and 315 were downregulated (Table 3 ). Out of the 331 characterized upregulated genes, the most enriched GO terms were those related to the TCA cycle, carbohydrate metabolism, as well as various metabolic processes. These points toward a shift in the metabolic profile, switching from the rapid but wasteful Crabtree overflow metabolism to a more energy conservative strategy utilizing the mitochondria, and storing excess carbon as glycogen and trehalose. Furthermore, the GO categories "response to oxidative stress" and "response to toxic substance" were also enriched. The 286 characterized downregulated genes were enriched for ribosome-/translationrelated processes as well as RNA metabolic processes. The enrichment analysis of the downregulated genes indicated a slowdown of the central cellular processes associated with adaptation to the stationary phase, i.e., quiescence (Coller, 2011), induced by the nitrogen depletion. The response is similar to what has been seen in previous studies (Boer et al., 2003), and even 

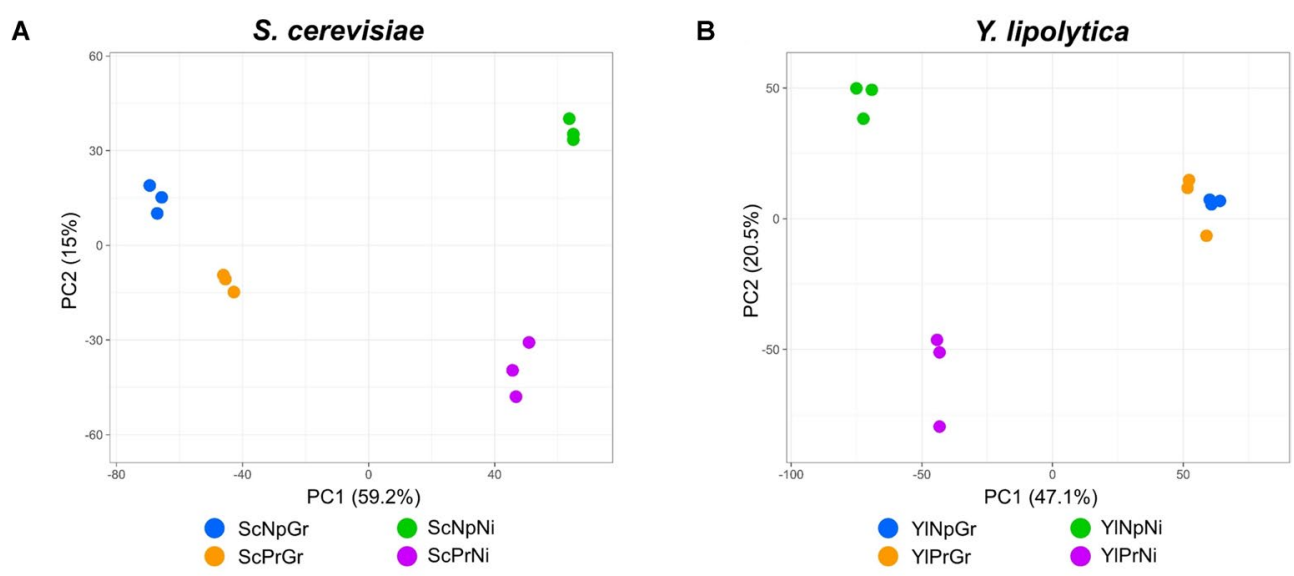

FIGURE 6 | Principal component analysis of transcriptomic data; PCA scatterplot showing S. cerevisiae RNA-seq samples projected onto the first two principal components of the data (A). PCA scatterplot showing Y. lipolytica RNA-seq samples projected onto the first two principal components of the data (B). Sc, Saccharomyces cerevisiae; YI, Yarrowia lipolytica; Pr, fatty alcohol producing; Np, non-producing; G, growth phase; Ni, nitrogen-depleted stationary phase.

though it is not revealed in the enrichment analysis, individual inspection of differentially expressed genes showed that the strains underwent transcriptional changes associated with the release of nitrogen catabolite repression (Daugherty et al., 1993; ter Schure et al., 2000) (Table S3). Nitrogen depletion triggered the upregulation of nitrogen transporters such as GAP1 (general amino acid permease, YKR039W) and PUT4 (proline permease, YOR348C), as well as the upregulation of enzymes involved in nitrogen metabolism such as DUR1,2 (urea amidolyase, YBR208C) and DAL1 (allantoinase, YIR027C).

The Y. lipolytica strains differentially expressed 631 genes in response to nitrogen depletion, out of which 500 were upregulated in nitrogen-depleted stationary phase, and 131 were downregulated. However, of the 500 upregulated genes 355 (71\%) were uncharacterized. Of the 145 characterized upregulated genes, GO terms relating to transporters were the only terms significantly enriched. A large part of these belonged to transporters for nitrogen-containing compounds such as ammonium, amino acids, oligopeptides, and urea. The upregulation of these transporters is likely the result of the alleviation of nitrogen catabolite repression imposed in the presence of ammonia, which is a logical biological response as the cell needs to find alternative nitrogen sources. Furthermore, considering the large increase in carboxylic acid secretion rate in response to nitrogen depletion (Table 2), carboxylic acid transporters were of particular interest. However, due to genes being associated with multiple GO terms, all the 11 carboxylic acid transporters predicted to be enriched were also annotated as amino acid transporters. Hence, the enrichment analysis didn't predict any known transporters of the TCA-associated organic acids. Of the 77 characterized down-regulated genes, only a single GO term, "sulfur compound metabolic process," was enriched. This does not appear to be related to sulfur-containing amino acids, but rather to the metabolism of other various sulfur-containing compounds possibly due to the unintended consequence of lowering (but not depleting) the extracellular sulfate concentration when depleting the media of nitrogen, which is added to the media in the form of ammonium sulfate.
In response to fatty alcohol production (producing strain $v s$. non-producing strain), the $S$. cerevisiae strain differentially expressed 24 genes, 21 of which were upregulated in the producing strain (Table 3). GO term enrichment analysis revealed that 9 out of the 17 characterized upregulated genes were associated with "cell wall organization or biogenesis." A previous meta-study (Arroyo et al., 2009) compared the transcriptional response to three different compounds (zymolase, congo red, and pneumocandins) triggering a cell wall stress response. Lowering the cutoff threshold of the differential gene expression from a 4 -fold to a 2-fold increase [same as used by the cell wall stress studies (Lagorce et al., 2003; Boorsma et al., 2004; García et al., 2004; Rodríguez-Peña et al., 2005) in the meta-analysis] revealed a significant overlap. Out of the 18 genes upregulated in all three cell wall stress conditions, 16 were found to be upregulated in response to FAR expression, indicating that the $S$. cerevisiae fatty alcohol-producing strain is experiencing cell wall stress. No other GO terms were enriched. Three genes were downregulated, all of which were uncharacterized.

The Y. lipolytica strains differentially expressed 215 genes in response to fatty alcohol production, out of which 60 genes were upregulated, and 155 were downregulated in the producing strain. GO term enrichment analysis singled out antibiotic catabolic process (especially formate catabolism) as a key factor among the 39 characterized genes upregulated during fatty alcohol production. Three of the 10 most upregulated characterized genes were formate dehydrogenases (YALI0_B22506g, YALI0_ B19976g, and YALI0_E14256g) with relatively high RNA abundance (CPM) and very low p-values (Supplementary Table S3), which further supports that the formate dehydrogenase upregulation is biologically significant. Formate dehydrogenases catalyze the reversible reaction between formate and carbon dioxide (formate $+\mathrm{NAD}^{+} \rightleftharpoons \mathrm{CO}_{2}+\mathrm{NADH}+\mathrm{H}^{+}$). Upregulation of formate dehydrogenases was also found to correlate with lipid accumulation in a recent study (Zhang et al., 2019), but the biological significance of the upregulation remains unclear. Genes annotated with the broad GO term "transporters" were 
TABLE 3 | GO term enrichment in differentially expressed genes. Go terms enriched more than 2.5-fold. Indented GO terms in brackets are a sub-group of the preceeding broader higher-level GO term. Differentially expressed genes described as condition 1 vs. condition 2, where upregulated signifies that condition 1 has a higher transcript abundance, and downregulated signifies that condition 2 has a higher transcript abundance. Uncharacterized genes consist of unclassified or unknown genes.

\section{S. cerevisiae: nitrogen depletion vs. growth}

Upregulated genes

Total: 401 genes, uncharacterized: 70 genes

GO biological process complete

Glyoxylate metabolic process (GO:0046487)

Oligopeptide transmembrane transport (GO:0035672)

Glutamate metabolic process (GO:0006536)

Antibiotic metabolic process (GO:0016999)

[Tricarboxylic acid cycle (GO:0006099)]

'De novo' protein folding (GO:0006458)

Ammonium transport (GO:0015696)

Carbohydrate biosynthetic process (GO:0016051)

Monosaccharide metabolic process (GO:0005996)

Carbohydrate catabolic process (GO:0016052)

Response to toxic substance (GO:0009636)

Monocarboxylic acid metabolic process (GO:0032787)

Carbohydrate metabolic process (GO:0005975)

Small molecule catabolic process (GO:0044282)

Response to oxidative stress (GO:0006979)

Cellular glucan metabolic process (GO:0006073)

Oxidation-reduction process (GO:0055114)

[Carboxylic acid catabolic process (GO:0046395)]

[Fatty acid beta-oxidation (GO:0006635)]

Response to heat (GO:0009408)

Drug metabolic process (GO:0017144)

Cellular response to external stimulus (GO:0071496)

Nucleobase-containing small molecule metabolic process

(GO:0055086)

[Nucleotide metabolic process (GO:0009117)]

Cofactor metabolic process (GO:0051186)

Coenzyme metabolic process (GO:0006732)

[Glycolytic process (GO:0006096)]

Downregulated genes

Total: 315 genes, uncharacterized: 29 genes

GO biological process complete

S-adenosylmethionine metabolic process (GO:0046500)

Regulation of establishment or maintenance of cell polarity

(GO:0032878)

Nucleobase biosynthetic process (GO:0046112)

DNA integration (GO:0015074)

rRNA-containing ribonucleoprotein complex export from nucleus

(GO:0071428)

Ribosomal small subunit biogenesis (GO:0042274)

DNA biosynthetic process (GO:0071897)

Amide biosynthetic process (GO:0043604)

[Translation (GO:0006412)]

Transposition, RNA-mediated (GO:0032197)

Ribosomal large subunit biogenesis (GO:0042273)

Cellular amino acid metabolic process (GO:0006520)

RNA phosphodiester bond hydrolysis (GO:0090501)

Cellular amide metabolic process (GO:0043603)

Nucleoside phosphate biosynthetic process (GO:1901293)

Gene count (331)
6
5
6
18
11
7
6
17
16
20
15
35
50
30
25
11
75
15
7
15
43
18
41
34
37
26
7

Expected
0.6
0.5
0.9
3.4
1.6
1.3
1.2
3.5
3.7
4.7
3.8
3.8
13.2
8
6.6
3
24.1
4.8
0.7
4.9
15.3
6.7
15.7
13.6
14
10.3
1.4

Fold enrichment
11
10.2
6.5
5.3
6.7
5.3
5.2
4.9
4.4
4.3
3.9
3.8
3.8
3.8
3.8
3.7
3.1
3.2
10.7
3.1
2.8
2.7
2.6

2.5
2.6
2.5
4.9

P-value

9.60E-05

$5.00 \mathrm{E}-04$

8.90E-04

8.70E-08

4.80E-06

8.50E-04

2.20E-03

4.80E-07

4.10E-06

3.10E-07

2.50E-05

1.50E-10

1.70E-14

5.00E-09

8.00E-08

4.50E-04

2.30E-17

2.20E-04

2.80E-05

2.70E-04

6.10E-09

3.30E-04

9.80E-08

3.00E-06

4.20E-07

5.00E-05

1.30E-03

$\begin{array}{cc}\text { Gene count (293) } & \text { Expected } \\ 5 & 0.3 \\ 4 & 0.3 \\ 7 & \\ 14 & 1.1 \\ 14 & 2.4 \\ & 3.1 \\ 28 & \\ 16 & 7 \\ 65 & 4.7 \\ 61 & 20.3 \\ 14 & 17.7 \\ 18 & 4.4 \\ 37 & 5.9 \\ 27 & 12.3 \\ 69 & 9.1 \\ 19 & 23.6 \\ & 7.4\end{array}$

$\begin{array}{cc}\begin{array}{c}\text { Fold enrichment } \\ \mathbf{1 4 . 8}\end{array} & \begin{array}{c}\text { P-value } \\ 1.20 \mathrm{E}-04\end{array} \\ \mathbf{1 1 . 8} & 1.10 \mathrm{E}-03 \\ & \\ \mathbf{6 . 6} & 2.70 \mathrm{E}-04 \\ \mathbf{5 . 8} & 8.80 \mathrm{E}-07 \\ \mathbf{4 . 6} & 8.90 \mathrm{E}-06 \\ & \\ \mathbf{4} & 3.60 \mathrm{E}-09 \\ \mathbf{3 . 4} & 4.90 \mathrm{E}-05 \\ \mathbf{3 . 2} & 6.40 \mathrm{E}-16 \\ \mathbf{3 . 4} & 3.10 \mathrm{E}-16 \\ \mathbf{3 . 2} & 3.00 \mathrm{E}-04 \\ \mathbf{3 . 1} & 6.90 \mathrm{E}-05 \\ \mathbf{3} & 1.10 \mathrm{E}-08 \\ \mathbf{3} & 1.70 \mathrm{E}-06 \\ \mathbf{2 . 9} & 4.30 \mathrm{E}-15 \\ \mathbf{2 . 6} & 3.10 \mathrm{E}-04\end{array}$

S. cerevisiae: producing vs. non-producing

\section{Upregulated genes}

Total: 21 genes, uncharacterized: four genes

GO biological process complete

Cell wall organization or biogenesis (GO:0071554)

Downregulated genes

Total: three genes, uncharacterized: three genes

GO biological process complete

None
Gene count (18)

9

Gene count (0)
Expected

0.8

Expected
Fold enrichment

11.0

Fold enrichment
P-value 
TABLE 3 | Continued

\section{Y. lipolytica: nitrogen depletion vs. growth}

\section{Upregulated genes}

Total: 500 genes, uncharacterized: 355 genes

GO biological process complete

Transmembrane transport (GO:0055085)

[Ammonium transmembrane transport (GO:0072488)]

[Carboxylic acid transmembrane transport (GO:1905039)]

[Amino acid transmembrane transport (GO:0003333)]

\section{Downregulated genes}

Total: 131 genes, uncharacterized: 54 genes

GO biological process complete

Sulfur compound metabolic process (GO:0006790)

Y. lipolytica producing vs. non-producing

Upregulated genes

Total: 60 genes, uncharacterized: 21 genes

GO biological process complete

Antibiotic catabolic process (GO:0017001)

[Formate catabolic process (GO:0042183)]

Downregulated genes

Total: 155 genes, uncharacterized: 79 genes

GO biological process complete

Transport (GO:0006810)

[Nitrogen compound transport (GO:0071705)]

[Carboxylic acid transmembrane transport (GO:1905039)]

[Amino acid transmembrane transport (GO:0003333)]

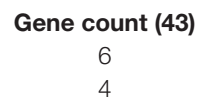

Gene count (121)

13

$\begin{array}{cc}\text { Eunt (315) } & \text { Expected } \\ 70 & 20.6 \\ 9 & 0.6 \\ 11 & 2.3 \\ 11 & 1.6\end{array}$

Expected

1.8

Fold enrichment
3.4
15.4
4.9
6.8

P-value

2.8E-18

$1.7 \mathrm{E}-07$

5.1E-05

3.6E-06

$\begin{array}{cc}\text { Fold enrichment } & \text { P-value } \\ \mathbf{4 2 . 8} & 1.57 \mathrm{E}-08 \\ \mathbf{6 0 . 0} & 1.59 \mathrm{E}-06\end{array}$

Expected
16.5
7.7
1.3
0.8

0.1

0.1

Gene count (120)
51
26
11
10

Fold enrichment
3.1
3.4
8.7
12.1

P-value

2.2E-11

6.8E-05

1.7E-04

4.3E-05 significantly enriched among the 76 downregulated characterized genes. Among the enriched transporters, the carboxylic acid transmembrane transporters were again of particular interest as downregulation of those genes could explain the decrease of byproduct secretion rate observed in response to fatty alcohol production (Table 2). However, due to genes being associated with multiple GO terms, out of the 11 transporters associated with carboxylic acids, 10 of them were amino acid transporters. The remaining transporter, YALI0_B19470g, was homologous to the S. cerevisiae transporter JEN1, annotated as a monocarboxylic acid transporter.

\section{DISCUSSION}

${ }^{13} \mathrm{C}$-flux analysis revealed fundamentally different metabolic profiles of the two yeast species S. cerevisiae and Y. lipolytica (Figure 5), which is in line with previous findings (Christen and Sauer, 2011). This has implications for engineering strategies. Given that fatty alcohols have a much higher energy content than glucose, energy efficiency and abundant reducing power (NADPH) will likely be key factors for high-performing cell factories. For S. cerevisiae, this means that removal of ethanol production and increased NADPH generation are necessary. Ethanol production is a major drain of carbon atoms and is energetically inefficient. As for NADPH generation, this could, for example, be achieved by re-routing more carbon through the pentose phosphate pathway; another solution might be to replace NAD-dependent glycolytic enzymes with NADP-dependent ones (Kildegaard et al., 2016). It is likely that fatty alcohol production in Y. lipolytica would also benefit from increased NADPH supply. An increased proportion of carbon going through the pentose phosphate pathway has previously been shown to improve lipid accumulation (Wasylenko et al., 2015). Efficient NADPH generation has also been achieved by expressing the NADPdependent glycerol-3-phosphate dehydrogenase (caGAPC), with either an NADH kinase ( $y$ lYEF1) or a cytosolic NADP-dependent malic enzyme (mcMCE2) (Qiao et al., 2017).

Transcriptomic analysis comparing producing and nonproducing strains of $S$. cerevisiae revealed a cell wall stress response. The cell wall stress response was indicated by both the GO term enrichment analysis (Table 3) as well as comparisons with previous studies (Arroyo et al., 2009). The cell wall-related upregulated genes have various functions. Crh1p and Crh2p are involved in cross-linking between the $(1,6)$ - $\beta$-glucan and chitin (Cabib et al., 2007). Slt2p is the MAPK (mitogen-activated protein kinase) responsible for triggering the cell wall stress response. Cwp1p, Pir2p, Pir3p, Ncw2p, Cis3p, Sed1p, Pst1p, and Ccw14p among others are covalently attached structural components of the cell wall. The reason for the triggering is unclear, but could conceivably be due to some kind of disturbance of the cell envelope caused by the produced fatty alcohols. It is interesting that no such stress response appears to be triggered in Y. lipolytica. The reasons for this difference are undetermined, but one hypothesis is that $Y$. lipolytica's lipid accumulating abilities allow it to incorporate the produced fatty alcohols into lipid bodies, reducing disturbance to the cell envelope such as the one observed in S. cerevisiae. In terms of design considerations, avoiding the toxic effects of fatty alcohols will likely be a part of any beneficial design. The cell wall stress response seen in $S$. cerevisiae might prove difficult to overcome by rational design; it is, however, possible that adaptive laboratory evolution selecting for increased growth in the presence of fatty alcohols could alleviate the problem. 
In Y. lipolytica, toxic effects were instead revealed in the nonproducing strain in the form of a growth defect (lower growth rate and final OD), which to some degree seems to be alleviated by the expression by the FAR (Figures 4B, D). The reasons for this phenomenon are unclear. However, we hypothesize that the growth defect might be due to the accumulation of medium chain fatty aldehydes due to a reaction between unsaturated long chain fatty acids and free radicals and/or molecular oxygen in $h f d$ negative cells as described in a previous study (Xu et al., 2017). The generation of free radicals might be worsened by the PEX10 knockout, which disrupts the peroxisome biogenesis and leads to oxidative stress (Van der Leij, 1992). Given that malFAR likely acts upon fatty aldehydes as a transient intermediate (Figure 1), it is possible that malFAR is also able to act upon medium chain fatty aldehydes, converting them into medium chain fatty alcohols, which possibly have a reduced toxic effect. At this point, this is mere speculation, and further experiments are needed to validate the hypothesis. Given the relocalization of peroxisomal matrix proteins to the cytoplasm as a result of the PEX10 knockout, it might prove a better approach to leave the peroxisome intact and all its enzymes enclosed. An alternative approach to PEX10 deletion is to prevent $\beta$-oxidation by knocking out the POX enzymes responsible for the first step in the $\beta$-oxidation cycle.

Nitrogen depletion appears to have some major drawbacks, such as reduced cellular and metabolic activities (Tables 2 and 3 ). This can be due to quiescence, which is a beneficial adaptation in a natural environment (Coller, 2011). Several studies on the cellular response to nitrogen limitation were made (Morin et al., 2011; Kerkhoven et al., 2016; Pomraning et al., 2016); though more research is needed to apply this knowledge to improve the cellular performance under the nitrogen limitation.

Although the metabolomics data doesn't directly reveal the limiting steps of fatty alcohol synthesis, it may help narrow down the possible options by the assumption that the limiting steps occur in metabolic pathways where data is lacking. The combination of the depleted acetyl-CoA pool (Figure 5), the low glucose uptake rate, and high organic acid secretion rate (Table 2) indicate that there are one or more limiting steps occurring upstream of acetyl-CoA with the glucose uptake and glucose phosphorylation and conversion to F6P/FBP as possible candidates. In the case of S. cerevisiae, the pyruvate/acetaldehyde/ acetate conversion is a potential target. The secretion profile (Table 2) of Y. lipolytica indicates that there are limiting steps following both pyruvate (pyruvate translocase and/or pyruvate dehydrogenase complex) and citrate (ATP-citrate lyase), or a possible competition with the plasma membrane-bound pyruvate and citrate transporters.

Furthermore, in Y. lipolytica, nitrogen depletion results in a significant increase in organic acid secretion, with the non-producing strain secreting $\sim 50 \%$ of the total carbon consumed in the form of organic acids. Knocking out organic acid transporters might help to limit this carbon loss. Based on the correlation of changing secretion rates with differential gene expression, and excluding amino acid transporters, a single carboxylic acid transporter (YALI0_B19470g) could be predicted and might be a contributor to the organic acid secretion. However, there are likely multiple other carboxylic transporters being upregulated among the currently unknown genes. A recent study (Zhang et al., 2019) indicates that lowering the $\mathrm{pH}$ of the media may shift Y. lipolytica from citrate secretion to lipid accumulation. Furthermore, the secretion of organic acids could also be reduced by expressing downstream enzymes using cytosolic organic acids as substrates, such as ATP-citrate lyase (citrate + ATP $\rightarrow$ acetyl-CoA + oxaloacetate) or pyruvate formate lyase (pyruvate $\rightarrow$ acetyl-CoA + formate); both of which has previously been shown to boost lipid accumulation (Xu et al., 2016). Overexpression of native Y. lipolytica ATP-citrate lyase resulted in modest improvements; however, heterologous ATP-citrate lyases might be proven more beneficial. Organic acid secretion might also be decreased by increasing the indirect pull from enzymes further downstream in the pathway (Figure 2). Overexpression of acetylCoA carboxylase (ACC1) and stearoyl-CoA desaturase (SGD1) in combination with diacylglyceride acyl-transferase (DGA1) has been shown to greatly boost lipid accumulation in Y. lipolytica (Qiao et al., 2015). In terms of fatty alcohol production, it's possible that the same strategy could be implemented, but replacing DGA1 with FAR.

This study describes a multi-omics analysis of the cellular and metabolic response to fatty alcohol production in two yeasts. It revealed cell wall stress response in fatty alcohol-producing $S$. cerevisiae. Furthermore, we have suggested designs that might aid in the engineering of fatty alcohol-producing cell factories.

\section{DATA AVAILABILITY}

The datasets generated for this study can be found in European Nucleotide Archive, PRJEB32352.

\section{AUTHOR CONTRIBUTIONS}

IB, JD, and $\mathrm{CH}$ conceived the study. JD performed the experiments. EM, CH, GW, and IB aided in troubleshooting and data interpretation. JD and DM performed method development, sampling and data processing of metabolomics data. JD and DW performed sample preparation and GCMS analysis for ${ }^{13} \mathrm{C}$-flux analysis. JD and UL performed data analysis of ${ }^{13} \mathrm{C}$-flux analysis data with the aid of BE. JD and CL performed data analysis of transcriptomic data. GCMS analysis of fatty alcohols was performed by JD, with the aid of H-LW. JD and IB wrote the manuscript with support from $\mathrm{CH}, \mathrm{EM}, \mathrm{GW}, \mathrm{UL}, \mathrm{BE}, \mathrm{MH}$, and LB. $\mathrm{IB}, \mathrm{LB}, \mathrm{MH}$, and $\mathrm{BE}$ supervised the project. $\mathrm{CH}$ and $\mathrm{GW}$ helped supervise the project.

\section{FUNDING}

This project has received funding from the European Union's Horizon 2020 research and innovation programme under the Marie Skłodowska-Curie grant agreement No 722287. IB acknowledges the financial support from the Novo Nordisk Foundation (Grant agreement NNF15OC0016592 and NNF10CC1016517) and from the European Research Council under the European Union's Horizon 2020 research and innovation programme (YEAST-TRANS project, Grant Agreement No 757384). MH, CL, and IB acknowledge the 
received funding from the European Union's Horizon 2020 research and innovation programme under grant agreement No. 760798 (OLEFINE project). LMB acknowledges funding by the Cluster of Excellence "The Fuel Science Center - Adaptive Conversion Systems for Renewable Energy and Carbon Sources," which is funded by the Excellence Initiative of the German federal and state governments to promote science and research at German universities.

\section{ACKNOWLEDGMENTS}

We thank Alexandra Hoffmeyer and Pannipa Pornpitakpong, for performing the RNA-seq analysis. We would also like to thank Dr. Hanne Bjerre Christensen and Dr. Lars Schrübbers for LC-MS and

\section{REFERENCES}

Arkin, A. P., Cottingham, R. W., Henry, C. S., Harris, N. L., Stevens, R. L., Maslov, S., et al. (2018). KBase: the United States department of energy systems biology knowledgebase. Nat. Biotechnol. 36, 566-569. doi: 10.1038/nbt.4163

Arroyo, J., Bermejo, C., García, R., and Rodríguez-Peña, J. M. (2009). Genomics in the detection of damage in microbial systems: cell wall stress in yeast. Clin. Microbiol. Infect. 15, 44-46. doi: 10.1111/j.1469-0691.2008.02676.x

Ashburner, M., Ball, C. A., Blake, J. A., Botstein, D., Butler, H., Cherry, J. M., et al. (2000). Gene Ontology: tool for the unification of biology. Nat. Genet. 25, 25-29. doi: $10.1038 / 75556$

Blank, L. M., Lehmbeck, F., and Sauer, U. (2005). Metabolic-flux and network analysis in fourteen Hemiascomycetous yeasts. FEMS Yeast Res. 5 (6-7), 545558. doi: 10.1016/j.femsyr.2004.09.008

Boer, V. M., de Winde, J. H., Pronk, J. T., and Piper, M. D. (2003). The genomewide transcriptional responses of Saccharomyces cerevisiae grown on glucose in aerobic chemostat cultures limited for carbon, nitrogen, phosphorus, or sulfur. J. Biol. Chem. 278 (5), 3265-3274. doi: 10.1074/jbc.M209759200

Bolger, A. M., Lohse, M., and Usadel, B. (2014). Trimmomatic: a flexible trimmer for Illumina sequence data. Bioinformatics 30 (15), 2114-2120. doi: 10.1093/ bioinformatics/btu170

Boorsma, A., de Nobel, H., ter Riet, B., Bargmann, B., Brul, S., Hellingwerf, K. J., et al. (2004). Characterization of the transcriptional response to cell wall stress in Saccharomyces cerevisiae. Yeast 21 (5), 413-427. doi: 10.1002/yea.1109

Borodina, I., Holkenbrink, C., Dam, M. I., and Löfstedt, C., (2018). Methods for producing fatty alcohols and derivatives thereof in yeast. WO2018109163A1. Geneva, Switzerland: World Intellectual Property Organization.

Buijs, N. A., Zhou, Y. J., Siewers, V., and Nielsen, J. (2015). Long-chain alkane production by the yeast Saccharomyces cerevisiae. Biotechnol. Bioeng. 112 (6), 1275-1279. doi: 10.1002/bit.25522

Cabib, E., Blanco, N., Grau, C., Rodríguez-Peña, J. M., and Arroyo, J. (2007). Crhlp and Crh2p are required for the cross-linking of chitin to $\beta(1-6)$ Glucan in the Saccharomyces cerevisiae cell wall. Mol. Microbiol. 63 (3), 921-935. doi: 10.1111/j.1365-2958.2006.05565.x

Christen, S., and Sauer, U. (2011). Intracellular characterization of aerobic glucose metabolism in seven yeast species by $13 \mathrm{C}$ flux analysis and metabolomics. FEMS Yeast Res. 11 (3), 263-272. doi: 10.1111/j.1567-1364.2010.00713.x

Coller, H. A. (2011). The essence of quiescence. Science 334 (6059), 1074-1075. doi: $10.1126 /$ science. 1216242

Daugherty, J. R., Rai, R., el Berry, H. M., and Cooper, T. G. (1993). Regulatory circuit for responses of nitrogen catabolic gene expression to the GLN3 and DAL80 proteins and nitrogen catabolite repression in Saccharomyces cerevisiae. J. Bacteriol. 175 (1), 64-73. doi: 10.1128/jb.175.1.64-73.1993

d'Espaux, L., Ghosh, A., Runguphan, W., Wehrs, M., Xu, F., Konzock, O., et al. (2017). Engineering high-level production of fatty alcohols by Saccharomyces cerevisiae from lignocellulosic feedstocks. Metab. Eng. 42, 115-125. doi: 10.1016/j. ymben.2017.06.004

Entian, K. D., and Kötter, P. (2007). Yeast genetic strain and plasmid collections. Method Microbiol. 36, 629-666. doi: 10.1016/S0580-9517(06)36025-4
GC-MS analysis. We thank Prof. Christer Löfstedt (Lund University) for analytical advice and access to GC-MS equipment for fatty alcohol analysis. We thank Prof. Volker Zickermann (Goethe-Universität) for the gift of Y. lipolytica GB20 strain and Dr. Peter Kötter, Johann Wolfgang Goethe University, Frankfurt, Germany for the gift of the S. cerevisiae CEN.PK113-7D strain. We would also like to thank Suresh Sudarsan and Marie Inger Dam for fruitful discussions.

\section{SUPPLEMENTARY MATERIAL}

The Supplementary Material for this article can be found online at: https://www.frontiersin.org/articles/10.3389/fgene.2019.00747/ full\#supplementary-material

Fillet, S., Gibert, J., Suárez, B., Lara, A., Ronchel, C., and Adrio, J. L. (2015). Fatty alcohols production by oleaginous yeast. J. Ind. Microbiol. Biotechnol. 42 (11), 1463-1472. doi: 10.1007/s10295-015-1674-x

Gaillardin, C. M., Charoy, V., and Heslot, H. (1973). A study of copulation, sporulation and meiotic segregation in Candida lipolytica. Arch. Mikrobiol. 92 (1), 69-83. doi: 10.1007/BF00409513

García, R., Bermejo, C., Grau, C., Pérez, R., Rodríguez-Peña, J. M., Francois, J., et al. (2004). The global transcriptional response to transient cell wall damage in Saccharomyces cerevisiae and its regulation by the cell integrity signaling pathway. J. Biol. Chem. 279 (15), 15183-15195. doi: 10.1074/ jbc. M312954200

Grand View Research, Inc. (2016). Fatty alcohols market by product (C6-C10, C11C14, C15-C22), by application (soaps \& detergents, personal care, lubricants, amines) expected to reach USD 7.51 billion by 2022. Available at: https://www. grandviewresearch.com/press-release/global-fatty-alcohols-market.

Holkenbrink, C., Dam, M. I., Kildegaard, K. R., Beder, J., Dahlin, J., Belda, D. D., et al. (2018). EasyCloneYALI: CRISPR/Cas9-based synthetic toolbox for engineering of the yeast Yarrowia lipolytica. Biotechnol. J. 13, 1700543. doi: 10.1002/biot.201700543

Iwama, R., Kobayashi, S., Ohta, A., Horiuchi, H., and Fukuda, R. (2014). Fatty aldehyde dehydrogenase multigene family involved in the assimilation of N-alkanes in Yarrowia lipolytica. J. Biol. Chem. 289 (48), 33275-33286. doi: 10.1074/jbc.M114.596890

Jessop-Fabre, M. M., Jakočiūnas, T., Stovicek, V., Dai, Z., Jensen, M. K., Keasling, J. D., et al. (2016). EasyClone-MarkerFree: a vector toolkit for marker-less integration of genes into Saccharomyces cerevisiae via CRISPRCas9. Biotechnol. J. 11 (8), 1110-1117. doi: 10.1002/biot.201600147

Kerkhoven, E. J., Pomraning, K. R., Baker, S. E., and Nielsen, J. (2016). Regulation of amino-acid metabolism controls flux to lipid accumulation in Yarrowia lipolytica. NPJ Syst. Biol. Appl. 2, 16005. doi: 10.1038/npjsba.2016.5

Kildegaard, K. R., Jensen, N. B., Schneider, K., Czarnotta, E., Özdemir, E., Klein, T., et al. (2016). Engineering and systems-level analysis of Saccharomyces cerevisiae for production of 3-hydroxypropionic acid via malonyl-CoA reductase-dependent pathway. Microb. Cell Fact. 15, 53. doi: 10.1186/s12934016-0451-5

Kim, D., Langmead, B., and Salzberg, S. L. (2015). HISAT: a fast spliced aligner with low memory requirements. Nat. Methods 12 (4), 357-360. doi: 10.1038/ nmeth.3317

Lagorce, A., Hauser, N. C., Labourdette, D., Rodriguez, C., Martin-Yken, H., Arroyo, J., et al. (2003). Genome-wide analysis of the response to cell wall mutations in the yeast Saccharomyces cerevisiae. J. Biol. Chem. 278 (22), 20345 20357. doi: 10.1074/jbc.M211604200

Liu, Y., Chen, S., Chen, J., Zhou, J., Wang, Y., Yang, M., et al. (2016). High production of fatty alcohols in Escherichia coli with fatty acid starvation. Microb. Cell Fact. 15 (1), 129. doi: 10.1186/s12934-016-0524-5

McCloskey, D., Gangoiti, J. A., Palsson, B. O., and Feist, A. M. (2015a). A PH and solvent optimized reverse-phase ion-pairing-LC-MS/MS method that leverages multiple scan-types for targeted absolute quantification of intracellular metabolites. Metabolomics 11 (5), 1338-1350. doi: 10.1007/s11306-015-0790-y 
McCloskey, D., Utrilla, J., Naviaux, R. K., Palsson, B. O., and Feist, A. M. (2015b). Fast Swinnex Filtration (FSF): a fast and robust sampling and extraction method suitable for metabolomics analysis of cultures grown in complex media. Metabolomics 11 (1), 198-209. doi: 10.1007/s11306-014-0686-2

McNeil, B. A., and Stuart, D. T. (2018). Optimization of C16 and C18 fatty alcohol production by an engineered strain of Lipomyces starkeyi. J. Ind. Microbiol. Biotechnol. 45 (1), 1-14. doi: 10.1007/s10295-017-1985-1

Mi, H., Huang, X., Muruganujan, A., Tang, H., Mills, C., Kang, D., et al. (2017). PANTHER version 11: expanded annotation data from gene ontology and reactome pathways, and data analysis tool enhancements. Nucleic Acids Res. 45 (D1), D183-D189. doi: 10.1093/nar/gkw1138

Morin, N., Cescut, J., Beopoulos, A., Lelandais, G., Le Berre, V., Uribelarrea, J.-L., et al. (2011). Transcriptomic analyses during the transition from biomass production to lipid accumulation in the oleaginous yeast Yarrowia lipolytica. PLOS ONE 6 (11), e27966. doi: 10.1371/journal.pone.0027966

Okonechnikov, K., Conesa, A., and García-Alcalde, F. (2016). Qualimap 2: advanced multi-sample quality control for high-throughput sequencing data. Bioinformatics 32 (2), 292-294. doi: 10.1093/bioinformatics/btv566

Pertea, M., Pertea, G. M., Antonescu, C. M., Chang, T.-C., Mendell, J. T., and Salzberg, S. L. (2015). StringTie enables improved reconstruction of a transcriptome from RNA-seq reads. Nat. Biotechnol. 33 (3), 290-295. doi: 10.1038/nbt.3122

Pomraning, K. R., Kim, Y.-M., Nicora, C. D., Chu, R. K., Bredeweg, E. L., Purvine, S. O., et al. (2016). Multi-omics analysis reveals regulators of the response to nitrogen limitation in Yarrowia lipolytica. BMC Genomics 17, 138. doi: 10.1186/s12864-016-2471-2

Poskar, C. H., Huege, J., Krach, C., Franke, M., Shachar-Hill, Y., and Junker, B. H. (2012). IMS2Flux - a high-throughput processing tool for stable isotope labeled mass spectrometric data used for metabolic flux analysis. BMC Bioinformatics 13 (1), 295. doi: 10.1186/1471-2105-13-295

Qiao, K., Imam Abidi, S. H., Liu, H., Zhang, H., Chakraborty, S., Watson, N., et al. (2015). Engineering lipid overproduction in the oleaginous yeast Yarrowia lipolytica. Metab. Eng. 29, 56-65. doi: 10.1016/j.ymben.2015.02.005

Qiao, K., Wasylenko, T. M., Zhou, K., Xu, P., and Stephanopoulos, G. (2017). Lipid production in Yarrowia lipolytica is maximized by engineering cytosolic redox metabolism. Nat. Biotechnol. 35, 173-177. doi: 10.1038/nbt.3763

Robinson, M. D., McCarthy, D. J., and Smyth, G. K. (2010). EdgeR: a bioconductor package for differential expression analysis of digital gene expression data. Bioinformatics 26 (1), 139-140. doi: 10.1093/bioinformatics/btp616

Rodríguez-Peña, J. M., Pérez-Díaz, R. M., Alvarez, S., Bermejo, C., García, R., Santiago, C., et al. (2005). The 'Yeast Cell Wall Chip'-a tool to analyse the regulation of cell wall biogenesis in Saccharomyces cerevisiae. Microbiology 151 (7), 2241-2249. doi: 10.1099/mic.0.27989-0

Schmitz, A., Ebert, B. E., and Blank, L. M., (2017). "GC-MS-based determination of mass isotopomer distributions for 13c-based metabolic flux analysis," in Hydrocarbon and Lipid Microbiology Protocols: Genetic, Genomic and System Analyses of Pure Cultures. Eds. T. J. McGenity, K. N. Timmis, and B. Nogales (Berlin, Heidelberg: Springer Berlin Heidelberg: Springer Protocols Handbooks), 223-243. doi: 10.1007/8623_2015_78

Shah, J., Arslan, E., Cirucci, J., O’Brien, J., and Moss, D. (2016). Comparison of oleo- vs petro-sourcing of fatty alcohols via cradle-to-gate life cycle assessment. J. Surfactants Deterg. 19 (6), 1333-1351. doi: 10.1007/s11743-016-1867-y
Tai, M., and Stephanopoulos, G. (2013). Engineering the push and pull of lipid biosynthesis in oleaginous yeast Yarrowia lipolytica for biofuel production. Metab. Eng. 15, 1-9. doi: 10.1016/j.ymben.2012.08.007

ter Schure, E. G., van Riel, N. A., and Verrips, C. T. (2000). The role of ammonia metabolism in nitrogen catabolite repression in Saccharomyces cerevisiae. FEMS Microbiol. Rev. 24 (1), 67-83. doi: 10.1111/j.1574-6976.2000.tb00533.x

The Gene Ontology Consortium (2017). Expansion of the gene ontology knowledgebase and resources. Nucleic Acids Res. 45 (D1), D331-D338. doi: 10.1093/nar/gkw1108

Thomas, P. D., Campbell, M. J., Kejariwal, A., Mi, H., Karlak, B., Daverman, R., et al. (2003). PANTHER: a library of protein families and subfamilies indexed by function. Genome Res. 13 (9), 2129-2141. doi: 10.1101/gr.772403

Van der Leij, I. (1992). Isolation of peroxisome assembly mutants from Saccharomyces cerevisiae with different morphologies using a novel positive selection procedure. J. Cell Biol. 119 (1), 153-162. doi: 10.1083/jcb.119.1.153

Wasylenko, T. M., and Stephanopoulos, G. (2015). Metabolomic and 13C-Metabolic flux analysis of a xylose-consuming Saccharomyces cerevisiae strain expressing xylose isomerase. Biotechnol. Bioeng. 112 (3), 470-483. doi: 10.1002/bit. 25447

Wasylenko, T. M., Ahn, W. S., and Stephanopoulos, G. (2015). The oxidative pentose phosphate pathway is the primary source of NADPH for lipid overproduction from glucose in Yarrowia lipolytica. Metab. Eng. 30, 27-39. doi: 10.1016/j.ymben.2015.02.007

Xu, P., Qiao, K., Ahn, W. S., and Stephanopoulos, G. (2016). Engineering Yarrowia lipolytica as a platform for synthesis of drop-in transportation fuels and oleochemicals. Proc. Natl. Acad. Sci. 113 (39), 10848-10853. doi: 10.1073/ pnas. 1607295113

Xu, P., Qiao, K., and Stephanopoulos, G. (2017). Engineering oxidative stress defense pathways to build a robust lipid production platform in Yarrowia lipolytica. Biotechnol. Bioeng. 114 (7), 1521-1530. doi: 10.1002/bit.26285

Young, J. D. (2014). INCA: a computational platform for isotopically nonstationary metabolic flux analysis. Bioinformatics 30 (9), 1333-1335. doi: 10.1093/bioinformatics/btu015

Zamboni, N., Fendt, S.-M., Rühl, M., and Sauer, U. (2009). 13C-based metabolic flux analysis. Nat. Protoc. 4 (6), 878-892. doi: 10.1038/nprot.2009.58

Zhang, S., Jagtap, S. S., Deewan, A., and Rao, C. V. (2019). PH selectively regulates citric acid and lipid production in Yarrowia lipolytica w29 during nitrogen-limited growth on glucose. J. Biotechnol. 290, 10-15. doi: 10.1016/j.jbiotec.2018.10.012

Conflict of Interest Statement: IB and $\mathrm{CH}$ have a financial interest in BioPhero ApS. The remaining authors declare that the research was conducted in the absence of any commercial or financial relationships that could be construed as a potential conflict of interest.

Copyright (C) 2019 Dahlin, Holkenbrink, Marella, Wang, Liebal, Lieven, Weber, McCloskey, Wang, Ebert, Herrgård, Blank and Borodina. This is an open-access article distributed under the terms of the Creative Commons Attribution License (CC $B Y)$. The use, distribution or reproduction in other forums is permitted, provided the original author(s) and the copyright owner(s) are credited and that the original publication in this journal is cited, in accordance with accepted academic practice. No use, distribution or reproduction is permitted which does not comply with these terms. 